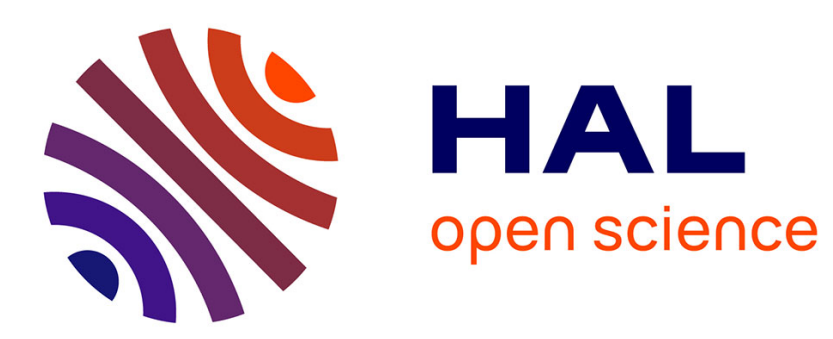

\title{
An inverse heat conduction problem with heat flux measurements
}

\author{
Tahar Loulou, Elaine Scott
}

\section{To cite this version:}

Tahar Loulou, Elaine Scott. An inverse heat conduction problem with heat flux measurements. International Journal for Numerical Methods in Engineering, 2006, 67 (11), pp.1587-1616. 10.1002/nme.1674 . hal-00401070

\section{HAL Id: hal-00401070 https://hal.science/hal-00401070}

Submitted on 25 Jun 2018

HAL is a multi-disciplinary open access archive for the deposit and dissemination of scientific research documents, whether they are published or not. The documents may come from teaching and research institutions in France or abroad, or from public or private research centers.
L'archive ouverte pluridisciplinaire HAL, est destinée au dépôt et à la diffusion de documents scientifiques de niveau recherche, publiés ou non, émanant des établissements d'enseignement et de recherche français ou étrangers, des laboratoires publics ou privés. 


\title{
An inverse heat conduction problem with heat flux measurements
}

\author{
Tahar Loulou ${ }^{1, *, \dagger}$ and Elaine P. Scott ${ }^{2, \dagger}$ \\ ${ }^{1}$ Univesité de Bretagne Sud, Centre de Recherche LET2E, 56321 Lorient, France \\ ${ }^{2}$ Department of Mechanical Engineering, Virginia Tech, Blacksburg, VA 24061-0238, U.S.A.
}

\begin{abstract}
Using heat flux measurements as additional information to solve inverse heat conduction problems was and is still rarely employed. Lot of disadvantages linked to heat flux measurement specificities (local disturbance, intrusive measurement, lack of knowledge and proficiency, etc.) make people prefer temperature measurements which are well documented and very widespread. Solving inverse heat conduction problems with heat flux measurements is quite different than the one which uses temperatures and need to be investigated deeply. In this work, this problem is approached through the solution of a bioengineering problem consisting in the development of a non-invasive blood perfusion probe. The effort here is focused on the development of a methodology for the estimation of timedependent blood perfusion from heat flux measurements. The physical probe incorporates a thin heat flux sensor, which is placed in contact with the tissue region where the perfusion is to be measured. The sensor records the heat flux due to an imposed thermal event, which is achieved by air flow. A one-dimensional mathematical model is used to simulate the thermal event occurring at the contact region holding between the probe and the tissue. A combined parameter and function estimation procedure is developed to estimate simultaneously time-dependent blood perfusion and thermal contact conductance between the probe and the tissue. The robustness of the method was demonstrated through several test cases using simulated data. The presented examples include various functional changes in the time evolution of blood perfusion. Results from this study have shown the feasibility of solving inverse problems with heat flux measurements and the two unknowns are estimated with no a priori information about their functional forms.
\end{abstract}

KEY WORDS: heat flux measurements; inverse thermal problem; conjugate gradient method; combined estimation; regularization

\footnotetext{
*Correspondence to: Tahar Loulou, Univesité de Bretagne Sud, Centre de Recherche LET2E, 56321 Lorient, France.

${ }^{\dagger}$ E-mail: tahar.loulou@univ-ubs.fr

*E-mail: scottep@vt.edu
} 


\section{INTRODUCTION}

A direct problem in the mathematical modelling of any physical system is to determine the response of a system, provided that the governing equations, the geometry of the domain of interest, the complete boundary and initial conditions, the material properties and the sources acting in the solution domain are given. When one or more conditions for solving the direct problem are partially or entirely unknown then an inverse problem may be formulated to estimate the unknowns from specified or measured system responses. The word estimate is used rather than determine or find because any values found through experimental data for unknown quantities are of necessity approximations to the true ones. It is well known that inverse problems are in general unstable in the sense that small measurement errors in the experimental data may amplify significantly the errors in their solutions [1]. As consequence, the inverse problems are ill-posed and hence they are more difficult to solve than direct problems.

In recent years, inverse problems have been extensively treated in several branches of sciences, such as solid mechanics, heat transfer, acoustics and electromagnetic scattering, electrical impedance tomography, hydrology, remote sensing, etc. The most common approach is to determine the optimal estimates of the model parameters or/and functions or/and boundary conditions by minimizing a selected measure-to-fit between experimental response of the system and the model computations.

The physical problem considered here involves a bioengineering problem which consists in characterizing the blood drainage in animal tissues. Blood perfusion is defined as the blood volume flow exchange through a given volume of tissue. It refers to the local, multidirectional blood flow through the capillaries and intercellular space of living tissue. Unlike the bulk flow of blood through the larger vessels, blood perfusion is considered to be a directionless quantity at the macroscopic level due to the convoluted nature of the pathways through which it moves. It is important for normal tissue physiology and is a part of the temperature regulatory system of the body. Changes in blood perfusion are also associated with a variety of pathologic processes.

The history of the measurement of local blood perfusion using thermal methods has been reviewed by several researchers (e.g. References [2,3]). Most perfusion measurement methods are based on the clearance of a thermal or chemical marker and require an invasive procedure. This not only disturbs the tissue to be measured, but causes discomfort and allows for the possibility of infection. This work is part of an ongoing research effort to develop a clinically practical, minimally invasive, blood perfusion measurement system. The overall effort includes the design and development of operational procedures for this system. The key elements of the system include a mechanism to impose a thermal event to the surface and a sensor which provides a time-resolved signal representing heat flux. Previous efforts have focused on estimating perfusion as a constant value. The objective of the work presented here is to develop an estimation procedure to simultaneously determine the time-dependent blood perfusion and thermal contact resistance using heat flux measurements from the probe.

A combined parameter and function estimation method based on the minimization of an objective function containing both computed and measured heat fluxes is used to estimate the time-dependent blood perfusion and the thermal conductance between the probe and the tissue. The minimization procedure is achieved by using the conjugate gradient method and the adjoint state equations; i.e. the gradient of the functional to be minimized is computed by the solution of the adjoint problem. Note that in the present research, heat flux rather than temperature 

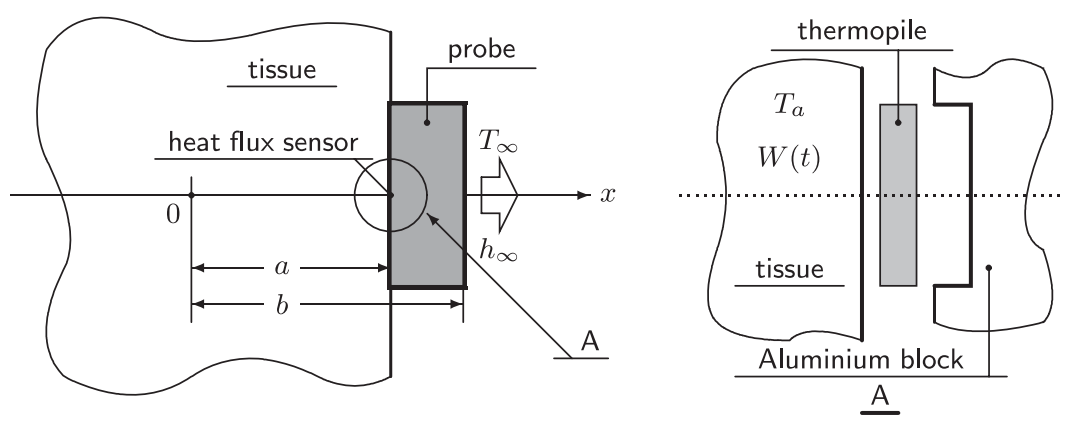

Figure 1. Bioprobe geometry, thermal model, and boundary conditions.

measurements are used in the objective function. This represents a new challenge in the sense that the theoretical background of solving an inverse problem with heat flux measurements has not been thoroughly investigated as for the existence, and the uniqueness of the solution [4-7]. The existence of a solution for such problem may be assured by physical reasoning and considerations. However, the uniqueness of the solution of inverse problems in general is more difficult to prove and can only be demonstrated for some special cases [8]. Mathematical background of such minimization problems, where the partial derivative of the state variable model is used instead of the state variable itself in the definition of the objective functional, is well treated in the control theory developed by Lions [9].

\section{PROBE DESIGN}

It is well known that the goals of a good heat transfer measurement is that it be non-intrusive to the system that being measured. The basic probe design, used in this study, is described in several references (e.g. References $[2,10,11]$ ), and is shown in Figure 1.

In this design, a combination of heat flux and temperature sensors forms the core of the probe. The heat flux is measured with a thermopile across a thin thermal resistance layer. The sensors are made with thin-film processes, resulting in small thermal capacitance, which allows them to accurately follow the change in heat flux and temperature. During operation, the front side of the probe is in continuous contact with the tissue, and the back side of the probe is cooled with small jets of air impinging normal to the surface. Velocities of less than $100 \mathrm{~m} / \mathrm{s}$ from a low pressure air supply at room temperature are sufficient to generate heat transfer coefficients in excess of $200 \mathrm{~W} / \mathrm{m}^{2} \mathrm{~K}$. The probe is small (less than $2.5 \mathrm{~cm}$ in diameter), simple, and lightweight which result in practical use. The theoretical background, the recommendations for a correct use and installation and the recent technological advances in heat flux measurements are presented and well detailed in Reference [12].

\section{DIRECT PROBLEM}

The mathematical model is based on the Pennes bioheat equation [13] to model the effect of blood perfusion on the tissue temperature in the tissue domain and on the transient heat equation 
to model the effect of cooling the probe region. A schematic representation of the model, including the probe and the tissue and given boundary conditions is shown in Figure 1. The heat transfer phenomenon is described mathematically below. For simplicity in demonstrating the estimation methodology, one-dimensional heat transfer is assumed; however, the estimation procedure is not limited to this simplified model, as the approach can be used with essentially any model. Thus, for the tissue (subscript t)

$$
\begin{aligned}
C_{\mathrm{t}} \frac{\partial T_{\mathrm{t}}(x, t)}{\partial t} & =k_{\mathrm{t}} \frac{\partial^{2} T_{\mathrm{t}}(x, t)}{\partial x^{2}}+W(t)\left[T_{\mathrm{a}}-T_{\mathrm{t}}(x, t)\right], \quad 0<x<a, \quad 0<t \leqslant t_{\mathrm{f}} \\
\frac{\partial T_{\mathrm{t}}(x, t)}{\partial x} & =0, \quad x=0, \quad t>0
\end{aligned}
$$

For the interface between the tissue and the probe

$$
\begin{aligned}
k_{\mathrm{t}} \frac{\partial T_{\mathrm{t}}(x, t)}{\partial x} & =k_{\mathrm{p}} \frac{\partial T_{\mathrm{p}}(x, t)}{\partial x}, \quad x=a, \quad 0<t \leqslant t_{\mathrm{f}} \\
-k_{\mathrm{t}} \frac{\partial T_{\mathrm{t}}(x, t)}{\partial x} & =H\left[T_{\mathrm{t}}(x, t)-T_{\mathrm{p}}(x, t)\right], \quad x=a, \quad 0<t \leqslant t_{\mathrm{f}}
\end{aligned}
$$

and for the probe (subscript $\mathrm{p}$ )

$$
\begin{aligned}
C_{\mathrm{p}} \frac{\partial T_{\mathrm{p}}(x, t)}{\partial t} & =k_{\mathrm{p}} \frac{\partial^{2} T_{\mathrm{p}}(x, t)}{\partial x^{2}}, \quad a<x<b, \quad 0<t \leqslant t_{\mathrm{f}} \\
-k_{\mathrm{p}} \frac{\partial T_{\mathrm{p}}(x, t)}{\partial x} & =h_{\infty}\left[T_{\mathrm{p}}(x, t)-T_{\infty}\right], \quad x=b, \quad 0<t \leqslant t_{\mathrm{f}}
\end{aligned}
$$

The initial conditions are described as

$$
\begin{array}{ll}
T_{\mathrm{t}}(x, 0)=T_{\mathrm{t}}^{i}, & 0 \leqslant x \leqslant a, \quad t=0 \\
T_{\mathrm{p}}(x, 0)=T_{\mathrm{p}}^{i}, & a \leqslant x \leqslant b, \quad t=0
\end{array}
$$

where $T_{\mathrm{t}}(x, t)$ and $T_{\mathrm{a}}$ are, respectively, the temperature in the tissue domain and the arterial temperature of the blood, and $T_{\mathrm{p}}(x, t)$ represents the temperature in the probe. The properties, $C_{i}=\rho_{i} c_{p_{i}}$ and $k_{i},(i=\mathrm{t}$ or $\mathrm{p})$ are, respectively, the volumetric specific heat and the thermal conductivity of the tissue and the probe. The volumetric blood perfusion rate is the product of the specific heat of the blood and the Pennes blood perfusion term; i.e. $W(t)=c_{p_{\mathrm{b}}} \omega(t)$. The heat transfer coefficient, $h_{\infty}$, is a result of the cooling air impingement on the back side of the probe, and a finite contact conductance, $H$, is assumed between the tissue and the probe, and is described by Equations (3) and (4). Finally, metabolic heat generation in the tissue region is neglected in this work.

In Equation (4), $H$ represents the thermal contact conductance which is the inverse of the contact resistance $(H=1 / R)$. In this problem under investigation, thermal contact conductance $H$ is assumed constant while the blood perfusion is a time-dependent function, $W(t)$. 
In the direct problem associated with the physical problem described above, the thermophysical properties $C_{i}, k_{i}(i=\mathrm{t}$ or $\mathrm{p})$, thermal conductance $H$, blood perfusion rate $W(t)$, external convection coefficient $h_{\infty}$, external air temperature $T_{\infty}$ as well as the arterial temperature $T_{\mathrm{a}}$, initial and boundary conditions, are known. The objective of the direct problem is then to determine the transient temperature field in the two regions; i.e. the tissue and the probe.

The solution of the direct problem given in Equations (1)-(8) is obtained numerically by using the finite difference method with a pure implicit scheme in time [14]. The numerical solution may show an oscillatory behaviour at the contact interface due to the significant difference between the material characteristics of the probe and the tissue. To overcome this instability, the Fourier number, resulting from the time and space subdivision and defined as

$$
F o=\frac{\alpha \Delta t}{(\Delta x)^{2}}
$$

should be the same for the two mediums, where $\Delta t$ and $\Delta x$ represent the time and space steps. Then, a stable numerical solution of the direct problem is obtained by setting

$$
F o=\frac{\alpha_{\mathrm{t}} \Delta t}{\left(\Delta x_{\mathrm{t}}\right)^{2}}=\frac{\alpha_{\mathrm{p}} \Delta t}{\left(\Delta x_{\mathrm{p}}\right)^{2}}
$$

for the two mediums. Thermal diffusivities $\alpha_{t}$ and $\alpha_{p}$ are set constants and imposed by the nature of the two regions in contact. The time evolution is often preferred to be the same for the two regions which exclude any choice on the time step $\Delta t$. Only the space grid size offers the possibility to be chosen so that the condition (10) will be satisfied. Practically as the probe has a thin thickness, its space grid $\Delta x_{\mathrm{p}}$ can be easily chosen and then $\Delta x_{\mathrm{t}}$ will be deduced as a function of $\Delta x_{\mathrm{p}}, \Delta t, \alpha_{\mathrm{t}}$ and $\alpha_{\mathrm{p}}$.

\section{INVERSE PROBLEM}

For the inverse problem considered here, the constant thermal conductance $H$ and the timedependent blood perfusion $W(t)$ are regarded as two unknowns, while the other quantities appearing in the formulation of the direct problem described above are assumed to be known precisely.

The additional information needed in the simultaneous estimation of the parameter $H$ and the function $W(t)$ is available from the readings of a heat flux sensor installed on the inner side of the probe (the sensor is a part of the probe) which is in imperfect thermal contact with the tissue region (Figure 1). Since it is desirable for the probe to be non-invasive, the heat flux measurement is performed outside the tissue domain.

In several methods, inverse problems are solved by minimizing a residual functional $J$ based on the ordinary least square norm and coupled with some stabilizing technique used in the iterative phase of the estimation procedure. The sum of the squared residuals between the measured data and the responses of a model simulating the physical problem under picture defines the least square norm in the space where the unknown quantities belong to. For continuous 
measured data, the residual functional is written as follows:

$$
J(H, W)=\int_{0}^{t_{\mathrm{f}}}\left[q_{\mathrm{c}}(a, t ; H, W)-q_{\mathrm{m}}(a, t)\right]^{2} \mathrm{~d} t
$$

where $q_{\mathrm{c}}(a, t ; H, W)$ and $q_{\mathrm{m}}(a, t)$ are, respectively, the computed and measured heat flux at the interface between the probe and the tissue while $t_{\mathrm{f}}$ denotes the final time. The computed heat flux is obtained from the solution of the direct problem by using estimates for the conductance $H$ and blood perfusion $W(t)$. This inverse problem deals with a combined parameter and function estimation. Note that this approach differs from common parameter and/or function estimation used to estimate thermal properties in the sense that another different type of measurements is used, namely heat flux, whereas typically temperatures are used.

As detailed in References [5,7,15], the solution of an inverse problem with the conjugate gradient method involves the following basic steps: with a given initial guess of the unknowns to be recovered, (a) solve the direct problem, (b) calculate the residual functional, (c) check the stopping criterion, if satisfied, stop; otherwise continue, (d) solve the adjoint problem, (e) compute the gradient equation and the corresponding descent direction, (f) solve the variation problem, (g) increment the unknowns and go back to step (a). Details associated with each of these steps follow, along with an outline of the computational algorithm.

To estimate the constant thermal conductance $H$ and the transient blood perfusion $W(t)$, we introduce the vector of unknowns $\mathbf{U}$ defined as

$$
\mathbf{U}^{\mathrm{T}}=\left[H, W_{1}, W_{2}, \ldots, W_{n_{\mathrm{t}}}\right]
$$

where the superscript $\mathrm{T}$ denotes the transpose, $W_{i}$ is the value of the blood perfusion at time $t_{i}$, i.e. $W_{i}=W\left(t_{i}\right)$, and $n_{\mathrm{t}}$ is the total number of time steps used in the solution of the direct problem. We recall that the time domain $\left[0, t_{\mathrm{f}}\right]$ is divided into equally spaced sub-intervals, equal to $\Delta t$, when solving the direct problem.

In this case, the total number of unknowns to be recovered by the solution of the inverse problem is $M=1+n_{\mathrm{t}}$. The minimization of the functional (11) by utilizing the conjugate gradient method is built as follows [16]:

$$
\mathbf{U}^{(s+1)}=\mathbf{U}^{(s)}+\gamma^{(s)} \mathbf{D}^{(s)}, \quad s=1,2, \ldots
$$

where the superscript $s$ is the iteration number, $\gamma^{s}$ is the descent parameter vector given by $\gamma^{\mathrm{T}}=\left[\gamma_{1}, \gamma_{2}\right]$, where the subscripts 1 and 2 correspond to $H$ and $W$. This is known as vectorial form of the descent parameter and is usually used when more than one unknown is to be recovered at once [17]. At each iteration, the two components $\gamma_{1}$ and $\gamma_{2}$ are solutions of the following linear algebraic system:

$$
\sum_{j=1}^{2} b_{j k} \gamma_{j}=c_{k} \quad \text { where } k=1,2
$$

The elements $b_{j k}$ and $c_{k}$ of the linear algebraic system are given by

$$
\begin{aligned}
& b_{j k}=\int_{0}^{t_{\mathrm{f}}} \Delta q_{k}(a, t) \Delta q_{j}(a, t) \mathrm{d} t \\
& c_{k}=-\int_{0}^{t_{\mathrm{f}}}\left[q_{\mathrm{c}}(a, t ; H, W)-q_{\mathrm{m}}(a, t)\right] \Delta q_{k}(a, t) \mathrm{d} t
\end{aligned}
$$


The two variables $\Delta q_{i}(a, t)(i=1,2)$ are the heat flux variation at the interface resulting from the solution of the two following variation problems $(i=1,2)$ :

$$
\begin{aligned}
C_{\mathrm{t}} \frac{\partial V_{\mathrm{t}}^{i}(x, t)}{\partial t} & =k_{\mathrm{t}} \frac{\partial^{2} V_{\mathrm{t}}^{i}(x, t)}{\partial x^{2}}-W(t) V_{\mathrm{t}}^{i}(x, t)+Y_{i}(x, t), \quad 0<x<a, \quad 0<t \leqslant t_{\mathrm{f}} \\
\frac{\partial V_{\mathrm{t}}^{i}(x, t)}{\partial x} & =0, \quad x=0, \quad 0<t \leqslant t_{\mathrm{f}} \\
k_{\mathrm{t}} \frac{\partial V_{\mathrm{t}}^{i}(x, t)}{\partial x} & =k_{\mathrm{p}} \frac{\partial V_{\mathrm{p}}^{i}(x, t)}{\partial x}, \quad x=a, \quad 0<t \leqslant t_{\mathrm{f}} \\
-k_{\mathrm{t}} \frac{\partial V_{\mathrm{t}}^{i}(x, t)}{\partial x} & =H\left[V_{\mathrm{t}}^{i}(x, t)-V_{\mathrm{p}}^{i}(x, t)\right]+Z_{i}(t), \quad x=a, \quad 0<t \leqslant t_{\mathrm{f}} \\
C_{\mathrm{p}} \frac{\partial V_{\mathrm{p}}^{i}(x, t)}{\partial t} & =k_{\mathrm{p}} \frac{\partial^{2} V_{\mathrm{p}}^{i}(x, t)}{\partial x^{2}}, \quad a<x<b, \quad 0<t \leqslant t_{\mathrm{f}} \\
-k_{\mathrm{p}} \frac{\partial V_{\mathrm{p}}^{i}(x, t)}{\partial x} & =h_{\infty} V_{\mathrm{p}}^{i}(x, t), \quad x=b, \quad t>0
\end{aligned}
$$

with the initial conditions

$$
\begin{aligned}
& V_{\mathrm{t}}^{i}(x, 0)=0, \quad 0 \leqslant x \leqslant a, \quad t=0 \\
& V_{\mathrm{p}}^{i}(x, 0)=0, \quad a \leqslant x \leqslant b, \quad t=0
\end{aligned}
$$

where the source terms $Y_{i}(x, t)$ and $Z_{i}(t)$ are given by

$$
\begin{gathered}
Y_{i}(x, t)= \begin{cases}0 & \text { for } i=1(H) \\
\Delta W(t)\left[T_{\mathrm{a}}-T_{\mathrm{t}}(x, t)\right] & \text { for } i=2(W(t))\end{cases} \\
Z_{i}(t)= \begin{cases}\Delta H\left[T_{\mathrm{t}}(a, t)-T_{\mathrm{p}}(a, t)\right] & \text { for } i=1(H) \\
0 & \text { for } i=2(W(t))\end{cases}
\end{gathered}
$$

In the iterative process expressed by Equation (13), $\mathbf{D}^{(s)}$ represents the descent direction vector which is given by

$$
\mathbf{D}^{(s)}=-\mathbf{J}^{\prime(s)}+\beta^{(s)} \mathbf{D}^{(s-1)}
$$

where $\mathbf{J}^{\prime}$ is the gradient vector of $J(\mathbf{U})$ and the parameter $\beta^{s}$ is given by

$$
\beta^{(s)}=\frac{\left\langle\mathbf{J}^{\prime(s)}-\mathbf{J}^{(s-1)}, \mathbf{J}^{\prime(s)}\right\rangle}{\left\langle\mathbf{J}^{\prime(s-1)}, \mathbf{J}^{\prime(s-1)}\right\rangle}, \quad \beta^{(0)}=0
$$


where $\langle$,$\rangle is the scalar product defined in the working space. The above expression is known$ as Polak-Ribière version of the conjugate gradient method [16]. Using the grid function representation of the blood perfusion $\left(W_{i}=W\left(t_{i}\right)\right)$, the gradient of the residual functional (11) is given by the vector

$$
\mathbf{J}^{\prime \mathrm{T}}=\left[J_{H}^{\prime}, J_{W_{1}}^{\prime}, \ldots, J_{W_{n_{\mathrm{t}}}}^{\prime}\right]
$$

For the simultaneous estimation of $H$ and $W(t)$ and assuming the grid function representation of $W(t)$, it can be shown that the components of the vector $\mathbf{J}^{\prime}$ have the following analytical expressions:

$$
\begin{aligned}
& J_{H}^{\prime}=\int_{0}^{t_{\mathrm{f}}}\left[T_{\mathrm{t}}(a, t)-T_{\mathrm{p}}(a, t)\right]\left[\left[q_{\mathrm{c}}(a, t ; H, W)-q_{\mathrm{m}}(a, t)\right]-\left[P_{\mathrm{t}}(a, t)-P_{\mathrm{p}}(a, t)\right]\right] \mathrm{d} t \\
& J_{W_{i}}^{\prime}=J_{W}^{\prime}\left(t_{i}\right)=\int_{0}^{a}\left[T_{\mathrm{a}}-T_{\mathrm{t}}\left(x, t_{i}\right)\right] P_{\mathrm{t}}\left(x, t_{i}\right) \mathrm{d} x, \quad i=1, \ldots, n_{\mathrm{t}}
\end{aligned}
$$

where $n_{\mathrm{t}}$ is the total number of time steps in the solution of the direct problem. The two gradients of the functional $J$ are derived and will be estimated in the space of squared integrable functions, named $L_{2}$. In the appendix we present the methodology used to derive analytically the gradient expressions. The variables $P_{\mathrm{t}}(x, t)$ and $P_{\mathrm{p}}(x, t)$ are solution of the adjoint problem which is defined by the following set of equations:

$$
\begin{aligned}
-C_{\mathrm{t}} \frac{\partial P_{\mathrm{t}}(x, t)}{\partial t} & =k_{\mathrm{t}} \frac{\partial^{2} P_{\mathrm{t}}(x, t)}{\partial x^{2}}-W(t) P_{\mathrm{t}}(x, t), \quad 0<x<a, \quad 0 \leqslant t<t_{\mathrm{f}} \\
\frac{\partial P_{\mathrm{t}}(x, t)}{\partial x} & =0, \quad x=0, \quad 0 \leqslant t<t_{\mathrm{f}} \\
k_{\mathrm{t}} \frac{\partial P_{\mathrm{t}}(x, t)}{\partial x} & =k_{\mathrm{p}} \frac{\partial P_{\mathrm{p}}(x, t)}{\partial x}, \quad x=a, \quad 0 \leqslant t<t_{\mathrm{f}} \\
-k_{\mathrm{t}} \frac{\partial P_{\mathrm{t}}(x, t)}{\partial x} & =H\left[P_{\mathrm{t}}(x, t)-P_{\mathrm{p}}(x, t)\right]-2 H\left[q_{\mathrm{c}}(x, t)-q_{\mathrm{m}}(x, t)\right], \quad x=a, \quad 0 \leqslant t<t_{\mathrm{f}} \\
-C_{\mathrm{p}} \frac{\partial P_{\mathrm{p}}(x, t)}{\partial t} & =k_{\mathrm{p}} \frac{\partial^{2} P_{\mathrm{p}}(x, t)}{\partial x^{2}}, \quad a<x<b, \quad 0 \leqslant t<t_{\mathrm{f}} \\
-k_{\mathrm{p}} \frac{\partial P_{\mathrm{p}}(x, t)}{\partial x} & =h_{\infty} P_{\mathrm{p}}(x, t), \quad x=b, \quad 0 \leqslant t<t_{\mathrm{f}}
\end{aligned}
$$

with the final conditions

$$
\begin{gathered}
P_{\mathrm{t}}(x, t)=0, \quad 0 \leqslant x \leqslant a, \quad t=t_{\mathrm{f}} \\
P_{\mathrm{p}}(x, t)=0, \quad a \leqslant x \leqslant b, \quad t=t_{\mathrm{f}}
\end{gathered}
$$


Parametrization of $W(t)$ : In some cases, the time evolution of the blood perfusion can be parametrized in the following way:

$$
W(t)=\sum_{i=1}^{m} w_{i} \mathscr{B}_{i}(t)
$$

The functions $\mathscr{B}_{i}(t)$ can be any trial functions (cosine, polynomials, B-splines, etc.) used to approximate the unknown function form of the blood perfusion $W(t)$. In this special case, the total number of unknowns to be recovered by the solution of the inverse problem becomes $M=1+m$ and the unknown vector $\mathbf{U}$ is given by

$$
\mathbf{U}^{\mathrm{T}}=\left[H, w_{1}, w_{2}, \ldots, w_{\mathrm{m}}\right]
$$

By introducing the parametric representation, one can show that the components of the gradient vector $\mathbf{J}^{\prime}$ with respect to those of blood perfusion change and will be expressed as

$$
J_{w_{i}}^{\prime}=\int_{0}^{t_{\mathrm{f}}} \int_{0}^{a} \mathscr{B}_{i}(t)\left[T_{\mathrm{a}}-T_{\mathrm{t}}(x, t)\right] P_{\mathrm{t}}(x, t) \mathrm{d} x \mathrm{~d} t, \quad i=1, \ldots, m
$$

while the gradient component with respect to $H, J_{H}^{\prime}$ remains the same as expressed by Equation (29). Also, the variation of $W(t)$ used in Equation (24) changes and will be given by

$$
\Delta W(t)=\sum_{i=1}^{m} d_{i} \mathscr{B}_{i}(t)
$$

where $d_{i}$ are the components of the descent vector $\mathbf{D}$ with respect to blood perfusion function. One beneficial point of the parametric representation is the reduction of the number of unknowns to be estimated. The parametric representation is recommended when the unknown $W(t)$ has a relatively smooth time evolution. In this case, the estimation procedure does not change in its algorithmic form but it takes place in the space of real parameters $\mathbb{R}^{m}$ (estimation of set of parameters) instead of function space.

Stopping criterion: In the absence of noise, the iterative process, Equation (13), is repeated until each component of the vector $\mathbf{U}$ satisfies the following stopping criteria:

$$
\left|\frac{u_{i}^{(s+1)}-u_{i}^{(s)}}{u_{i}^{(s+1)}}\right| \leqslant \varepsilon_{1}, \quad i=1, \ldots, M
$$

where $\varepsilon_{1}$ is a small number $\left(10^{-4}-10^{-6}\right)$. In the event that the input heat flux values are given with errors, the iterative process is stopped in accordance with the residual criterion or discrepancy principle [7], i.e. upon fulfilment of the following condition:

$$
J(\mathbf{U}) \leqslant \varepsilon_{2}
$$

where $\varepsilon_{2}$ is given by

$$
\varepsilon_{2}=\int_{0}^{t_{\mathrm{f}}} \sigma^{2} \mathrm{~d} t=\sigma^{2} t_{\mathrm{f}}
$$


It represents the integrated error of the measured data and having a constant standard deviation $\sigma$. Many iterative methods exhibit a self-regularizing property in the sense that early termination of the iterative process has a regularizing effect [18]. In the iterative regularization method, the iteration index $s$ plays the role of the regularizing parameter $\alpha$ used in Tikhonov's method [19], and the stopping rule $\left(J(\mathbf{U}) \leqslant \varepsilon_{2}\right)$ plays the role of the parameter selection method. Physically speaking, the solution is assumed to be sufficiently accurate and close to the exact one when the difference between measured and estimated heat flux is of the order of magnitude of the measurement errors.

Minimization algorithm: By using a given initial guess for $H^{(0)}$ and $W^{(0)}(t)$, the minimization procedure can be implemented following this scheme:

1. Solve the direct problem given in Equations (1)-(8).

2. Knowing the computed and measured heat flux $q_{\mathrm{c}}(a, t)$ and $q_{\mathrm{m}}(a, t)$, solve the adjoint problem given in Equations (31)-(38).

3. Knowing the temperature field $T_{i}(x, t)$ and the adjoint variable $P_{i}(x, t)$, compute the components of the gradient vector: Equations (28)-(30).

4. Knowing the gradient components, compute the descent direction by using Equations (26) and (27).

5. Knowing the two source terms $Y_{i}(x, t)$ and $Z_{i}(t)$, solve twice the variation problem given in Equations (16)-(23), i.e. the first time with $Y_{1}(x, t)$ and $Z_{1}(t)$, the second time with $Y_{2}(x, t)$ and $Z_{2}(t)$.

6. Knowing the computed heat flux $q_{\mathrm{c}}(a, t)$, the measured heat flux $q_{\mathrm{m}}(a, t)$, and the two heat fluxes variations $\Delta q_{1}(a, t)$ and $\Delta q_{2}(a, t)$, compute the components of descent vector: Equations (14) and (15).

7. Increment the two unknowns $H$ and $W(t)$ as shown in Equation (11).

8. Check convergence using formula (43) or (44). If satisfied stop, otherwise go back to step (1).

At each iteration, we must solve three problems, the direct, the adjoint and the variation problem (twice in this special case). As these problems are similar, we can use the same numerical algorithm to perform this resolution. The three different problems are solved numerically using the finite difference method [14].

\section{SIMULATED EXPERIENCE}

In this section, we describe the simulated experiments used to evaluate the inverse methodology. These simulated experiments are presented in the form of five test cases, described below. The input data used in the direct problem are shown in Table I. The subscripts $(t)$ and $(p)$ designate, respectively, the tissue and the probe.

The direct, variation, and adjoint problems are solved with finite-difference method with an implicit discretization in time. The spatial domain was subdivided with $n_{x}=n_{x 1}+n_{x 2}$, while $n_{\mathrm{t}}$ time steps are used to advance the solution from 0 to $t_{\mathrm{f}}$. The following data were taken for all the test cases presented here

$$
\begin{aligned}
a & =0.01 \mathrm{~m}, \quad b-a=0.005 \mathrm{~m}, \quad t_{\mathrm{f}}=30 \mathrm{~s}, \quad \varepsilon_{1}=10^{-5} \\
F o_{\mathrm{t}} & =F o_{\mathrm{p}}=100, \quad n_{\mathrm{t}}=121, \quad n_{x 1}=523, \quad n_{x 2}=11
\end{aligned}
$$


Table I. Input parameters and properties for direct problem.

\begin{tabular}{lccc}
\hline Component & Parameter & Value & Units \\
\hline \multirow{4}{*}{ Tissue } & $C_{t}$ & 3770 & $\mathrm{~J} / \mathrm{m}^{3}{ }^{\circ} \mathrm{C}$ \\
& $k_{t}$ & 0.50 & $\mathrm{~W} / \mathrm{m}^{\circ} \mathrm{C}$ \\
& $c_{p_{\mathrm{b}}}$ & 3770 & $\mathrm{~J} / \mathrm{kg}^{\circ} \mathrm{C}$ \\
& $\omega(t)$ & $1-100$ & $\mathrm{~kg} / \mathrm{m}^{3} \mathrm{~s}$ \\
Probe & $T_{a}$ & 37 & ${ }^{\circ} \mathrm{C}$ \\
& $T_{t}^{i}$ & 32 & ${ }^{\circ} \mathrm{C}$ \\
\multirow{4}{*}{ Boundary } & $C_{p}$ & 1768900 & $\mathrm{~J} / \mathrm{m}^{3}{ }^{\circ} \mathrm{C}$ \\
& $k_{p}$ & 177 & $\mathrm{~W} / \mathrm{m}^{\circ} \mathrm{C}$ \\
& $T_{p}^{i}$ & 20 & ${ }^{\circ} \mathrm{C}$ \\
& $h_{\infty}$ & 250 & $\mathrm{~W} / \mathrm{m}^{2}{ }^{\circ} \mathrm{C}$ \\
& $T_{\infty}$ & 20 & ${ }^{\circ} \mathrm{C}$ \\
\hline
\end{tabular}

Note: The blood perfusion rate $\omega(t)$ is assumed to vary from 1 to 100 according to the functions associated with each of the test cases.

Simulated measurements: The simulated measurements of heat flux are obtained from the solution of the direct problem at the sensor location, by using a priori prescribed values for the unknown parameter and function to be recovered simultaneously. Then, exact value of thermal contact conductance $H$ is taken as $1000 \mathrm{~W} / \mathrm{m}^{2} \mathrm{~K}$ for all the examples shown in this study. The time evolution of blood perfusion is given by

$$
W(t)=c_{p_{\mathrm{b}}} \omega(t)
$$

where $c_{p_{\mathrm{b}}}$ is the constant specific heat of the blood, and $\omega(t)$ is a function characterizing the time dependence of the blood perfusion.

The solution of the direct problem by using the known parameter and function provides the exact (errorless) heat flux measurements $q_{x}\left(a, t_{i}\right), i=1, \ldots, n_{\mathrm{t}}$. Note that real measurements generally contain errors. Therefore, measurements containing random errors are simulated by adding a white noise (error term) to $q_{x}\left(a, t_{i}\right)$ in the form

$$
q_{\mathrm{m}}\left(a, t_{i}\right)=q_{x}\left(a, t_{i}\right)+\varpi \sigma
$$

where $q_{\mathrm{m}}\left(t_{i}\right)$ is the simulated measurements of heat flux, $q_{x}\left(t_{i}\right)$ is the exact heat flux (errorless), $\sigma$ is the standard deviation of the measurement errors, $\varpi$ is a random variable with normal distribution, zero mean and unitary standard deviation. For the $99 \%$ confidence level the random number $\varpi$ belongs to the interval ] $-2.576<\varpi<+2.576[$. This variable is generated with the subroutine DRRNOR of the IMSL library [20]. The use of simulated measurements obtained in such a way can be very helpful and gives insights into understanding the physics of the problem under picture as for the model adequacy, the solution stability with respect to errors, and the optimal design of the experiment. In this case, the inverse problem solution procedure shall be able to estimate the values a priori prescribed for the two unknowns.

Inverse problem solution: We now consider the inverse problem of estimating the parameter $H$ and the function $W(t)$ by the conjugate gradient method. In all presented test cases, 
the initial guesses for the unknown parameter $H$ and for the unknown function $W(t)$ were taken as

$$
H^{(0)}=100, \quad W^{(0)}(t)=0
$$

Estimation error: To quantify the relative error of the estimation procedure, the following definitions are introduced:

$$
\varepsilon_{H}=\left|\frac{H-\bar{H}}{\bar{H}}\right| \times 100 \%
$$

is the computation error for thermal conductance $H$, and

$$
\varepsilon_{\omega}=\frac{\int_{0}^{t_{\mathrm{f}}}[\bar{\omega}(t)-\omega(t)]^{2} \mathrm{~d} t}{\int_{0}^{t_{\mathrm{f}}}[\bar{\omega}(t)]^{2} \mathrm{~d} t} \times 100 \%
$$

is the estimation error for blood perfusion function. The over-bar designates the exact parameter or function under hand. Another measure of the accuracy of recovered perfusion, since the true value is known, is the root-mean-square error which is defined as

$$
\varrho_{\omega}=\left[\frac{1}{n_{\mathrm{t}}} \sum_{i=1}^{n_{\mathrm{t}}}\left[\omega_{x}\left(t_{i}\right)-\omega\left(t_{i}\right)\right]^{2}\right]^{1 / 2}
$$

where $\omega\left(t_{i}\right)$ is the estimated blood perfusion function at time $t_{i}, \omega_{x}\left(t_{i}\right)$ is the exact blood perfusion used to generate the simulated heat flux measurements at time $t_{i}$, and $n_{\mathrm{t}}$ is the total number of measurements.

Since the gradient equation relative to blood perfusion, see expressions (37)-(38), is null at the final time, the initial guess used for $\omega\left(t_{\mathrm{f}}\right)$ is never changed by the iterative procedure, generating instabilities on the solution in the neighbourhood of final time $t_{\mathrm{f}}$. One approach to overcome such difficulties is to consider a parametric representation of the unknown function to be estimated, i.e. the blood perfusion $\omega(t)$. This is accomplished through the use of Equation (39) where the basis functions are chosen as spline functions $\mathscr{B}_{i}^{\kappa}(t) . \kappa$ designates the order of the spline which can take the value 1, for a constant spline function, 2 for a linear spline function, 3 for a parabolic spline function, or 4 for a cubic spline function. More details on the derivation and the use of spline functions can be found in Reference [21].

Four test cases, representing different functional variations for perfusion, used to evaluate the estimation procedure are analysed below. Two analysis of the simulated data are discussed for each test case. In the first analysis the blood perfusion and thermal conductance are estimated using exact data while the second analysis is performed using noisy data. In each analysis, blood perfusion is recovered in its two representations (i.e. parametric and non-parametric form), and a brief comparison between them is presented. Parametric form means that the blood perfusion is approximated by means of Equation (39). Non-parametric form designates the grid function representation; i.e. we have as many blood perfusion unknowns as time nodes (see Equation (12)).

Table II summarizes the results obtained for the simultaneous estimation of thermal conductance and blood perfusion, estimation error, computing time, and number of iterations, as applied to the cases shown in Figures 2-10. All computations were performed on a Pentium 
Table II. Results obtained from simulated test cases, with two measurement level errors $\sigma=0$ and $\sigma=120$.

\begin{tabular}{|c|c|c|c|c|c|c|c|}
\hline $\begin{array}{l}\text { Data } \\
\text { error }\end{array}$ & $\begin{array}{l}\text { Test } \\
\text { case }\end{array}$ & $\begin{array}{l}\text { Representation } \\
\text { form }\end{array}$ & $\begin{array}{c}\text { Estimated } \\
H\left(\mathrm{~W} / \mathrm{m}^{2} \mathrm{~K}\right)\end{array}$ & $\begin{array}{l}\varepsilon_{H} \\
(\%)\end{array}$ & $\begin{array}{c}\varepsilon_{\omega} \\
(\%)\end{array}$ & $\underset{\left(\mathrm{kg} / \mathrm{m}^{3} \mathrm{~s}\right)}{\varrho_{\omega}}$ & $\begin{array}{c}\text { Iterations } \\
\text { number }\end{array}$ \\
\hline \multirow{8}{*}{$\sigma=0$} & \multirow[b]{2}{*}{1} & Parametric & 1000.00 & 0.00 & 0.00 & 0.01 & 221 \\
\hline & & Grid function & 999.84 & 0.01 & 0.99 & 4.60 & 42 \\
\hline & \multirow[b]{2}{*}{2} & Parametric & 999.99 & 0.00 & 0.00 & 0.01 & 41 \\
\hline & & Grid function & 1000.02 & 0.02 & 0.25 & 4.57 & 43 \\
\hline & \multirow[b]{2}{*}{3} & Parametric & 1000.00 & 0.00 & 0.00 & 0.06 & 119 \\
\hline & & Grid function & 999.86 & 0.01 & 0.42 & 3.74 & 26 \\
\hline & \multirow[b]{2}{*}{4} & Parametric & 1000.11 & 0.01 & 3.62 & 4.43 & 20 \\
\hline & & Grid function & 999.99 & 0.00 & 0.00 & 0.11 & 78 \\
\hline \multirow{8}{*}{$\sigma=120$} & \multirow[b]{2}{*}{1} & Parametric & 1003.17 & 0.31 & 0.99 & 3.49 & 13 \\
\hline & & Grid function & 997.28 & 0.27 & 5.38 & 8.31 & 8 \\
\hline & \multirow[b]{2}{*}{2} & Parametric & 1003.21 & 0.32 & 0.62 & 5.20 & 7 \\
\hline & & Grid function & 975.26 & 2.47 & 4.21 & 14.53 & 5 \\
\hline & \multirow[b]{2}{*}{3} & Parametric & 1001.41 & 0.14 & 0.50 & 3.11 & 12 \\
\hline & & Grid function & 995.20 & 0.47 & 3.22 & 7.94 & 6 \\
\hline & \multirow[b]{2}{*}{4} & Parametric & 999.37 & 0.06 & 4.80 & 5.09 & 9 \\
\hline & & Grid function & 998.77 & 0.12 & 10.19 & 7.43 & 9 \\
\hline
\end{tabular}

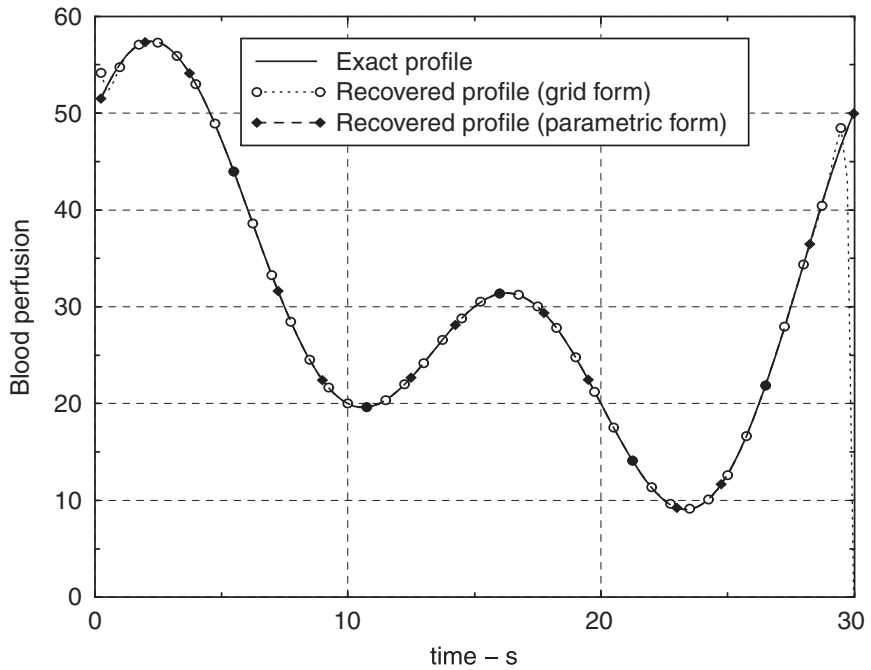

Figure 2. Results of test case 1, obtained with errorless data. Comparison between exact and recovered blood perfusion profile obtained with grid and parametric representation. Parametric form is built with $\kappa=4$ and $m=16$.

Platform. Two different levels of measurements errors considered for numerical analysis included $\sigma=0$ (errorless) and $\sigma=0.01 q_{\max }$, where $q_{\max }$ is the maximum measured heat flux at the interface between the tissue and the probe. 


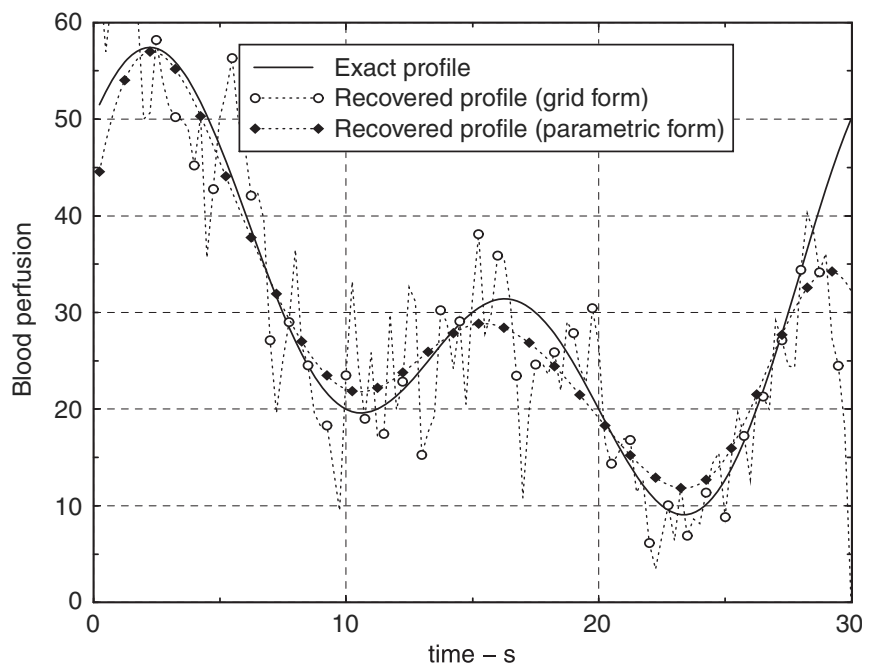

Figure 3. Results of test case 1, performed with noisy data. Comparison between exact and recovered blood perfusion profile obtained with grid and parametric representations. Parametric form of blood perfusion is built with $\kappa=4$ and $m=9$.

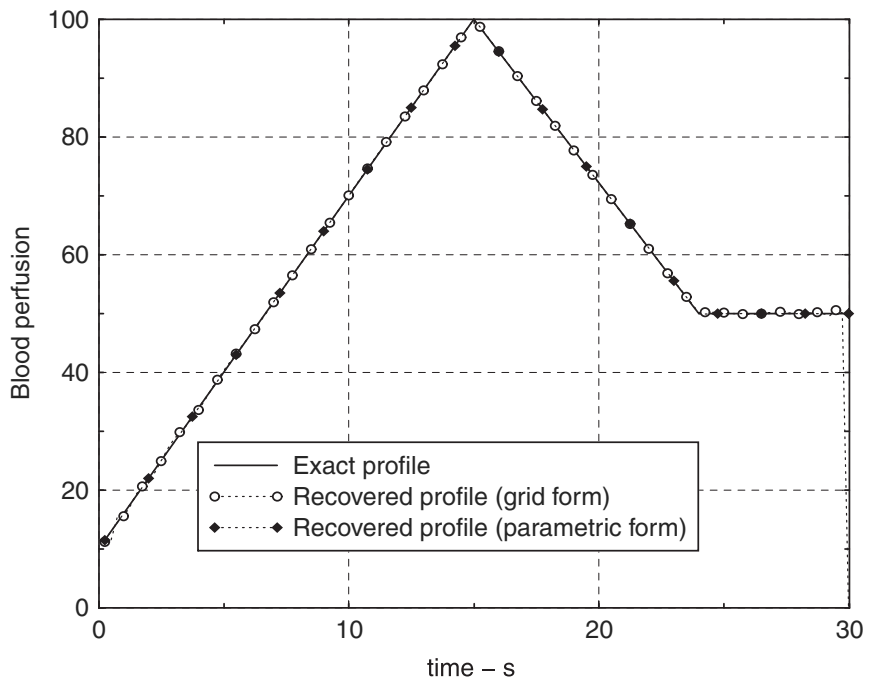

Figure 4. Test case 2: Comparison between exact and recovered profile of blood perfusion obtained with errorless data and for both its representation: grid and parametric.

Test case 1: Smooth variation.

In the first test, the time evolution of blood perfusion follows a sinus function and was assumed to vary according to the following expression:

$$
W(t)=c_{p_{\mathrm{b}}} W_{o}\left[3+\sin \left(3 \pi t / t_{\mathrm{f}}\right)+\cos \left(3 \pi t / t_{\mathrm{f}}\right)+\sin \left(6 \pi t / t_{\mathrm{f}}\right)+\cos \left(6 \pi t / t_{\mathrm{f}}\right)\right]
$$




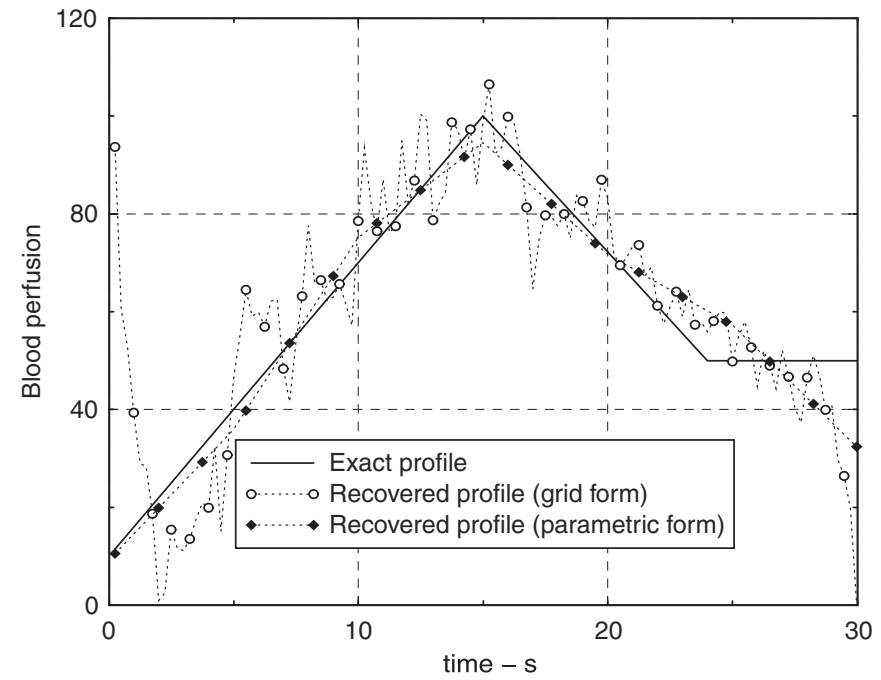

Figure 5. Test case 2: Comparison between exact and recovered profile of blood perfusion obtained with noisy data and for both representation of blood perfusion: grid and parametric forms.

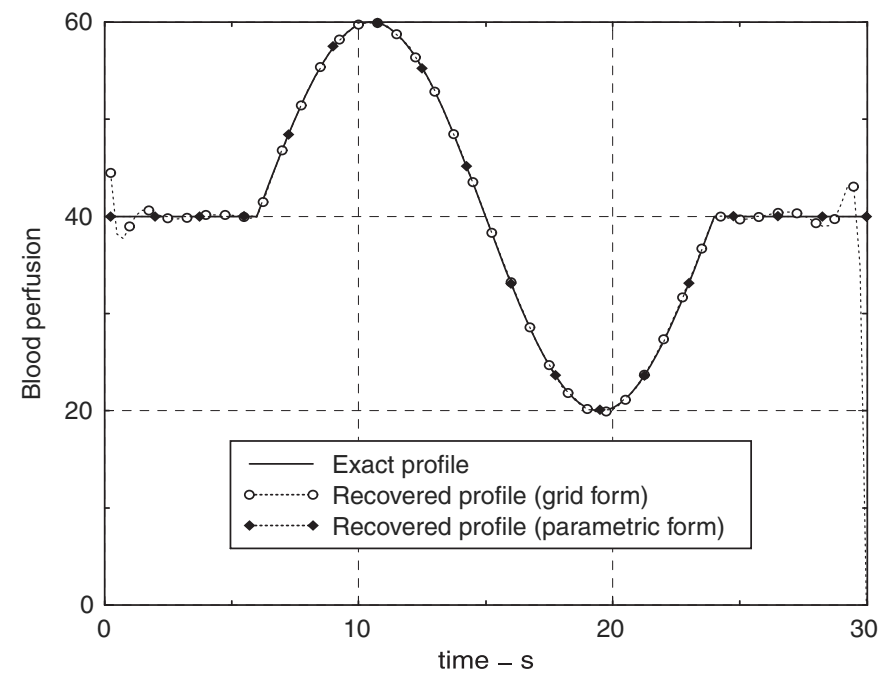

Figure 6. Test case 3: Comparison between exact and recovered profile of blood perfusion obtained with errorless data and for both its representation: grid and parametric forms.

The parameter $W_{o}$ was taken as 10 . This case represents the easiest test. The smoothness of the perfusion function gives more flexibility to the estimation procedure and does not present any difficulty in its parametrization choice. Few parabolic or cubic basis functions are sufficient to represent correctly the time variation. 


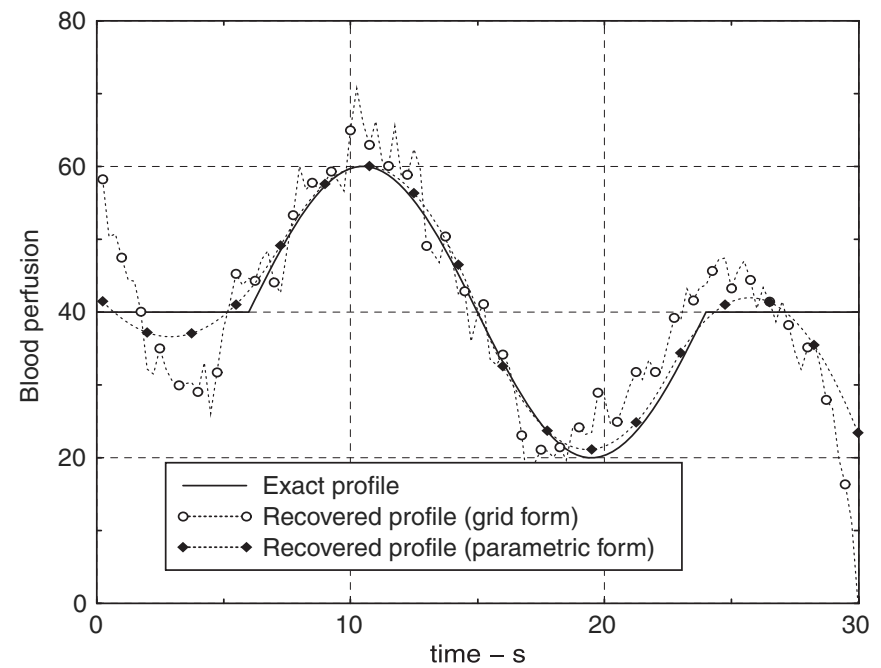

Figure 7. Test case 3: Comparison between exact and recovered profile of blood perfusion obtained with noisy data and for both its representation: grid and parametric forms.

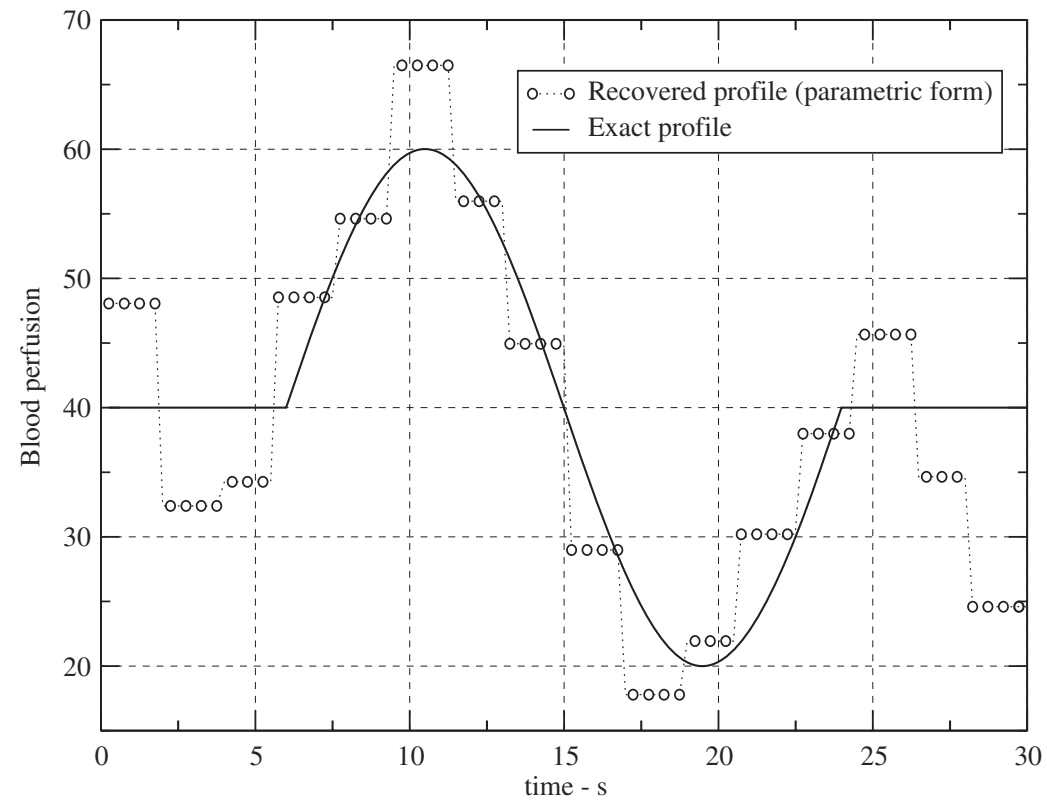

Figure 8. Test case 3: Comparison between exact and recovered profile of blood perfusion obtained with noisy data and parametric form. 


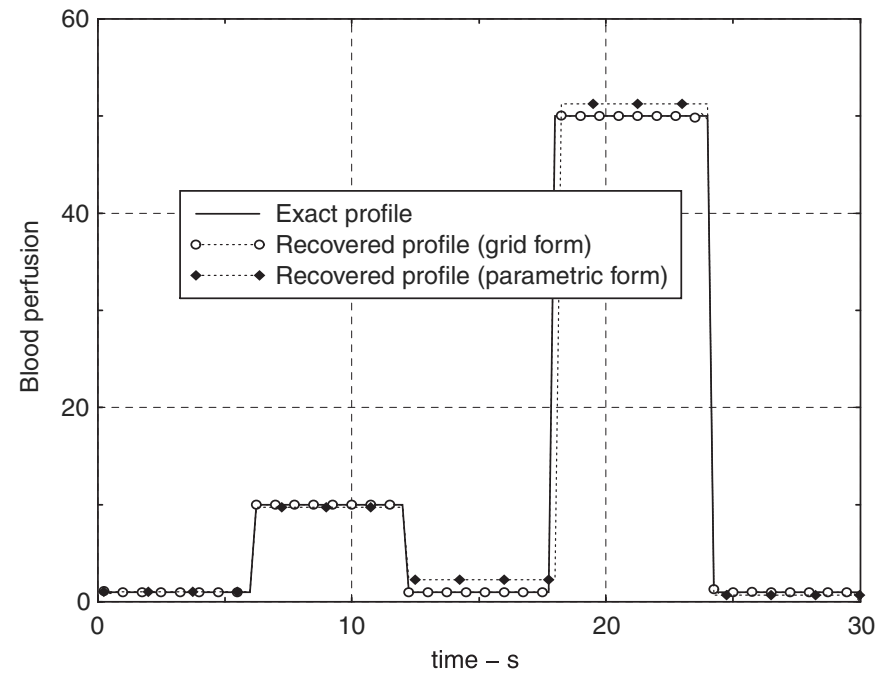

Figure 9. Estimated blood perfusion profile for test case 4 in its two representations: parametric and non-parametric forms. The presented results are obtained with exact data.

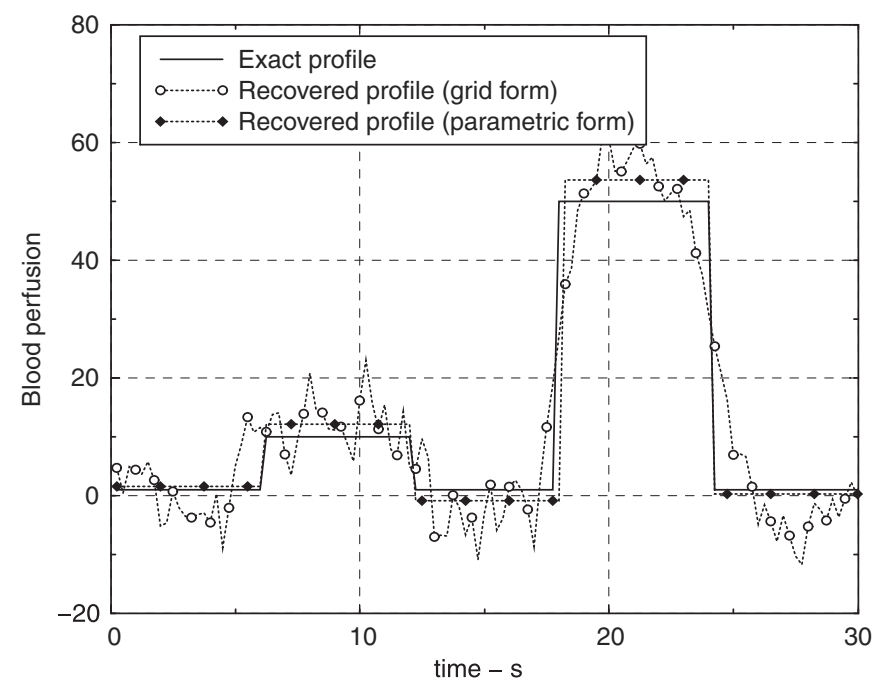

Figure 10. Comparison between the reconstructed blood perfusion profiles with the exact one obtained with and without its parametrization form. The presented results are obtained with noised data.

Figure 2 displays the recovered blood perfusion profile obtained with errorless data for both representations, i.e. non-parametric and parametric form. As expected the grid representation (non-parametric form) presents some deficiency at the beginning and at the end due to the null 
end-condition of gradient equation. Otherwise the estimation is very good over the remaining time interval. The estimation results obtained with a parametric form of blood perfusion are in excellent agreement with exact profile over all the time interval. As reported in Table II, the estimation error, for both unknowns are good in general and better when the blood perfusion is parametrized. The estimation error is less than $0.01 \%$ for both unknowns. Despite a relatively high iteration number, which results in a greater computation time, the recovered $H$ and $W(t)$ function coincide precisely with their exact values.

Figure 3 shows the results of the inverse analysis for the first test case with a measurement error of $\sigma=120 \mathrm{~W} / \mathrm{m}^{2}$. This standard deviation represent $1 \%$ error with respect to the maximum heat flux at the interface. The results of perfusion when it is estimated in the non-parametric form exhibits relatively high noise randomly scattered about the exact profile. The parametric representation reduces drastically this noise and the recovered profile presents approximately the same time evolution as the exact one. Numerical simulations have that the best results are obtained when the perfusion is parametrized with cubic spline function $(\kappa=4)$ and using nine basis functions $(m=9)$. By using parametric form the estimation error of perfusion is reduced five times while the estimation error of thermal conductance remains of the same order of magnitude. Greater noise level cannot be checked here because the inverse problem solution tends to show instabilities that have approximately the amplitude of test case variation itself.

Test case 2: Linear and constant variation.

The time-dependent blood perfusion for test case two is a combination of triangular ramp and a short constant part. Analytically, the second test case is described by

$$
W(t)=c_{p_{\mathrm{b}}} W_{o} \times \begin{cases}0.60 t+1 & \text { for } 0<t \leqslant t_{\mathrm{f}} / 2 \\ -0.55 t+18.33 & \text { for } t_{\mathrm{f}} / 2<t \leqslant 3 t_{\mathrm{f}} / 4 \\ 5 & \text { for } 3 t_{\mathrm{f}} / 5<t \leqslant t_{\mathrm{f}}\end{cases}
$$

The parameter $W_{o}$ is chosen as 10 . Looking to Figure 4 where the results are displayed, no difficulties to underline in the estimation procedure when it is applied with errorless data. The estimation results for both unknowns are nearly exact with a straight advantage to those obtained with the parametric representation of blood perfusion. As shown in Table II, the estimation error is null when the estimation is performed with parametric form of blood perfusion. In this case, a combination of linear spline functions, $\kappa=2$, was found to be the best approximation with an optimal number $m$ of 11 basis functions. With this choice, the estimation error was less than $1 \%$ for both unknowns, i.e. thermal conductance and blood perfusion. By choosing $\kappa=1$ (constant function) and $m=121$ as key parameters for the basis functions in Equation (39), which means we have as many basis functions as time nodes, we get precisely the same results obtained without using parametric form of the blood perfusion. We should mention here that the choice of $\kappa=1$, the basis functions are reduced to set of constants.

Figure 5 illustrates the effects of the same standard deviation $\sigma=120 \mathrm{~W} / \mathrm{m}^{2}$, used in the first test case, on the accuracy of the estimates for both representation of blood perfusion. The results obtained with non-parametric form of perfusion show the same perturbations observed in the first test case. These perturbations are randomly scattered around the exact profile which means that the estimation procedure is able to recover the general tendency of the time variation of blood perfusion but fails to recover it precisely due to the inherent ill-posed character of inverse problems. The error of recovered thermal conductance is very acceptable. An optimal 
number of seven $(m=7)$ linear spline function $(\kappa=2)$ was used to parametrize blood perfusion in this case.

Test case 3: Constant and sinusoidal variation.

As a third example, the blood perfusion is assumed to be constant over two short time intervals at the beginning and at the end and to have a sinus time variation between them, i.e. over the remaining time interval. This variation is assumed to vary in the following form:

$$
W(t)=c_{p_{\mathrm{b}}} W_{o} \times \begin{cases}2 & \text { for } 0<t \leqslant t_{\mathrm{f}} / 5 \\ 2+\sin \left[\frac{2 \pi\left(t-t_{\mathrm{f}} / 5\right)}{3 t_{\mathrm{f}} / 5}\right] & \text { for } t_{\mathrm{f}} / 5<t \leqslant 4 t_{\mathrm{f}} / 5 \\ 2 & \text { for } 4 t_{\mathrm{f}} / 5<t \leqslant t_{\mathrm{f}}\end{cases}
$$

The parameter $W_{o}$ is taken as 10 . Here again the estimation procedure does not encounter any difficulty in recovering precisely the two unknowns when the errorless data are used. With the parametric form of perfusion, the estimation error is null for both unknowns. Due to the discontinuities of the first derivative of this function at the connecting points with its constant parts, the best parametric form was found by considering constant or linear spline function as basic function in Equation (39). This above statement is still valid when errorless measurements are used in the solution of the inverse problem. The presented results in Figure 6 were obtained with 31 linear spline functions, i.e. $\kappa=2$, and $m=31$. As expected, the recovered blood perfusion in parametric form is more accurate than the one obtained without parametric representation (grid form). Here again the iteration number and computation time increase with the parametric form.

The recovered blood perfusion profile in its two forms (parametric and non-parametric) is displayed in Figure 7. These results were obtained with noisy data having the same standard deviation $\sigma=120 \mathrm{~W} / \mathrm{m}^{2}$. The best parametric form was obtained by considering six parabolic spline functions $(\kappa=3, m=6)$. With the parametric form, the estimation error is reduced to less than $1 \%$ for both unknowns while the iteration number is doubled for this test case. The right parametrization of perfusion was difficult to find in this special case due to the two inflexion points present in the profile. Acceptable results, shown in Figure 8, were obtained with 16 constant spline functions too.

Test case 4: Double step variation.

The last example deals with a function presenting double step which represents a very strict test for the inverse analysis. The time-dependent blood perfusion for a double step function was assumed to vary in the form

$$
W(t)=c_{p_{\mathrm{b}}} W_{o} \times \begin{cases}1 / 10 & \text { for } 0<t \leqslant t_{\mathrm{f}} / 5 \\ 1 & \text { for } t_{\mathrm{f}} / 5<t \leqslant 2 t_{\mathrm{f}} / 5 \\ 1 / 10 & \text { for } 2 t_{\mathrm{f}} / 5<t \leqslant 3 t_{\mathrm{f}} / 5 \\ 10 & \text { for } 3 t_{\mathrm{f}} / 5<t \leqslant 4 t_{\mathrm{f}} / 5 \\ 1 / 10 & \text { for } 4 t_{\mathrm{f}} / 5<t \leqslant t_{\mathrm{f}}\end{cases}
$$

where $W_{o}$ was taken as 10 . This example represents the hardest test case, among the presented tests, in the sense that it simulates two abrupt changes, with different amplitude, in the blood 


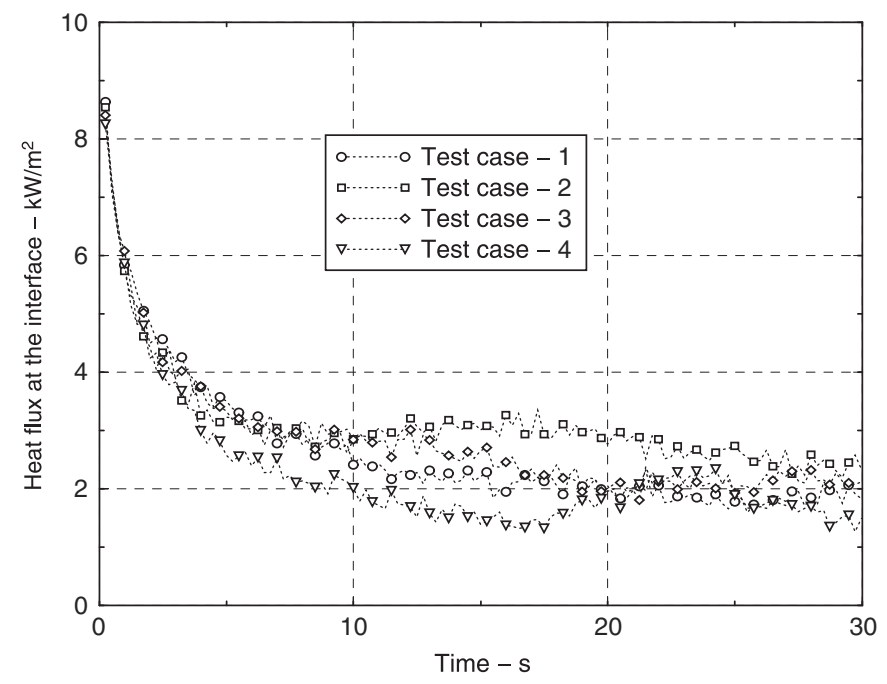

Figure 11. Comparison of the time evolution of the measured heat flux at the interface between the probe and the tissue for the four presented test cases.

perfusion during the total experimental period. Figure 9 compares the results of the inverse solution for both the representation of blood perfusion, parametric and non-parametric form obtained with errorless data. Here again the estimation procedure works well in recovering simultaneously the two unknowns. As displayed in Table II, both estimation error are relatively small. Contrary to the previous examples, the estimated results with non-parametric form of perfusion are better. Indeed the estimation was nearly perfect for both $H$ and $W(t)$ with an important iteration number. With the presence of two discontinuities in the profile, the parametrization was difficult to accomplish. The use of parabolic functions and higher order will smooth the profile, especially at the sharp corners, and good results cannot be obtained in this case. This is why the parametrization will be performed with constant functions rather than linear functions.

Estimated perfusion profiles in parametric form and non-parametric form with the use of noisy data are displayed in Figure 10. The results for this test without parametrization, follow the two pulses but cannot predict correctly the sharp corners. The global time evolution is recovered with low accuracy around the abrupt changes. Five constant spline functions $(\kappa=1, m=5)$ were found to be the best approximation for blood perfusion. The choice of $m=5$ is suggested by the fact that the time evolution of perfusion presents 5 equally spaced time intervals over which the perfusion has a constant value. In other words, this is an exploration of an a priori information on the perfusion behaviour.

Figure 11 shows measured heat flux at the interface between the probe and the tissue for the four test cases investigated in this study. Based on the initial temperatures in the probe and the tissue, the maximum value of this heat flux is about $12000 \mathrm{~W} / \mathrm{m}^{2}$ at time $t>0$. (This value is not shown in Figure 11.) The heat flux decreases drastically in short time period which represents approximately $15 \%$ of the total experimental time. Each displayed heat flux profile 
was obtained by adding a white noise of a standard deviation of $\sigma=120 \mathrm{~W} / \mathrm{m}^{2}$ to the exact profile. This standard deviation represents $1 \%$ measurement error based on the maximum heat flux. Numerical simulation show that, as the error increases, the accuracy of the prediction decreases and the results tend to be unworkable.

In all presented results, the thermal contact conductance $H$ was recovered with high accuracy. This is probably due to the relatively small deviation in the initial guess of $H$. In this study, lower values than $85 \mathrm{~W} / \mathrm{m}^{2} \mathrm{~K}$ as an initial guess of $H$ were checked and they do not give convergence of the estimation procedure. We should mention here that, with a null initial guess, the recovered blood perfusion profile is still very acceptable. That means the estimation of blood perfusion can be performed without using any a priori information on its time evolution.

A general comparison of different results of Table II reveals the following conclusions:

1. The CPU times for parametric form are larger than those for the grid function representation but are still acceptable. The evaluation of the spline functions during each iteration is at the origin of the increase.

2. The iteration numbers are higher with parametrization than with the grid function representation. Also the iteration numbers are widely reduced when the inverse analysis is performed with noisy data and they appear to be of the same order of value for all the presented examples.

3. With errorless data and the parametric form of blood perfusion the root mean square of perfusion is reduced four hundreds times (400), except for the fourth test case. The smallest root mean square is observed with smooth function simulating perfusion.

4. By using exact data and the different spline function representations, the estimation error is null for all the test cases except the fourth test. This estimation error increases when noisy data are used with the parametrization. The highest error is observed with test case four for both measurement levels.

The four presented test cases represent extremely difficult time evolutions of the blood perfusion. These cases were chosen to verify the robustness of the estimation procedure. As expected, it is easier to recover a continuous and smooth function, such as the one given by Equation (51), than a function containing discontinuities in the first derivative, like the triangular variation given by Equation (52), or like the constant-sinus variation given by Equation (53), or like the double step variation given by Equation (54). Physically when the tissue is under treatment, the temperature changes (increase or decrease) and induces a distention or shrinkage of the vessel ramifications. As a result there is a smooth variation of blood perfusion. Also, the same statement can be applied to exclude any abrupt change in blood perfusion.

\section{CONCLUDING REMARKS}

An inverse heat conduction problem which uses heat flux measurements has been formulated and solved. The minimization procedure is conducted by reducing the least square norm between experimental measured heat flux and its corresponding calculated values from a mathematical model. This approach differs from common parameter and/or function estimation methods which uses temperature measurements to solve inverse problems. The minimization problem stated in this paper is solved iteratively by the conjugate gradient method coupled to the discrepancy 
principle as stopping criterion. The gradient of the least square norm to be minimized is obtained by means of the adjoint state equations.

As demonstrating example a bioengineering problem is investigated. Indeed, a new combined experimental and numerical procedure is presented for the simultaneous estimation of timedependent blood perfusion and thermal conductance using a non-invasive air-cooled blood perfusion probe.

To the best knowledge of the authors this is the first time where the conjugate gradient method using the adjoint state equation approach is used with heat flux measurement instead of temperatures to estimate simultaneously two unknowns (one parameter and one time-dependent function). The developed estimation procedure was applied successfully to several shapes of blood perfusion. A comparison of the recovered data with the exact model showed good agreement. The obtained results underline the feasibility of the procedure and its capabilities to recover simultaneously thermal conductance and blood perfusion without using any a priori information on the unknown transient blood perfusion.

In some cases, the estimated blood perfusion at the beginning and the end of the experiment did not agree well with the prescribed one used in generating the simulated heat flux. This deficiency is due to the inherent problem of the null end-condition in the adjoint problem which generates instabilities on the solution. Efforts are currently underway to address this problem by considering the appropriate parametric representation of the unknown blood perfusion. The optimal choice of parameters needed in the parametrization and the reconstruction of blood perfusion as a temperature function will be examined.

\section{APPENDIX A}

\section{A.1. Inverse problem statement}

The inverse problem under picture deals with the estimation of two unknowns (one parameter, one function) in the space of square integrable functions $L_{2}$. In the variational formulation, the inverse problem consists in minimizing the discrepancy between computed and measured values of heat flux expressed by the functional $J(H, W)$ with respect to desired functions $H$ and $W(t)$. The minimization procedure is repeated iteratively until an acceptable level of closeness between measurements and computations is reached. The measured heat flux $q_{\mathrm{m}}(a, t)$, cannot be any arbitrary time-dependent function. Indeed, it is strongly connected with the two unknowns, $H$ and $W(t)$ via the direct heat conduction problem. In other words, we look for the minimum of the cost function $J$, while the dynamic equations (1)-(8) describing the space and temporal evolution of the temperature, act as a set of strong constraints, i.e. they are fulfilled exactly (equality constraints). Within the domain $[0, b] \times\left[0, t_{\mathrm{f}}\right]$, the evolution of the model variables $T_{\mathrm{t}}(x, t)$ and $T_{\mathrm{p}}(x, t)$ are fully controlled by the unknowns $H$ and $W(t)$. The problem is to find these two unknowns such that the least square distance between model response and experimental data, i.e. cost function $J$, is minimized and the constraints fully satisfied. This problem is well known in the field of the control theory under the name of minimization with constraints.

To minimize the residual functional with respect to desired unknowns, we use unconstrained gradient type methods, in particular, the conjugate gradient method [16]. To compute the residual functional gradient, we use a method based on the theory of calculus of variations. 
Thus, the initial problem of minimization with constraints is transformed to another problem of minimization without constraints by introducing the Lagrange functional associated to the direct problem. Then the stationary conditions of Lagrange functional are studied to derive the analytical form of the gradient. To develop this approach, we need to introduce some basic calculus of variations.

\section{A.2. Direct problem in variations}

Finding the minimum of the residual functional by gradient-type methods requires the derivation of its gradient which can be accomplished through the two following steps based on the calculus of variations. First, the computation of its variation when the unknowns will change with a small amount, and second, inspection of the conditions under which we obtain a stationary point (saddle point) for the associated Lagrange functional.

To compute the direct problem in variations (or the variation problem), the unknowns $H$ and $W(t)$ are perturbed simultaneously by an amount denoted $\varepsilon \Delta H$ and $\varepsilon \Delta W(t)$, that is

$$
\begin{gathered}
H_{\varepsilon}=H+\varepsilon \Delta H \\
W_{\varepsilon}(t)=W(t)+\varepsilon \Delta W(t)
\end{gathered}
$$

Thence, the temperature undergoes the variations $\varepsilon \Delta T_{\mathrm{t}}(x, t)$ and $\varepsilon \Delta T_{\mathrm{p}}(x, t)$ respectively, which can expressed as

$$
\begin{aligned}
& T_{\mathrm{t} \varepsilon}(x, t)=T_{\mathrm{t}}(x, t)+\varepsilon \Delta T_{\mathrm{t}}(x, t)=T_{\mathrm{t}}(x, t)+\varepsilon V_{\mathrm{t}}(x, t) \\
& T_{\mathrm{p} \varepsilon}(x, t)=T_{\mathrm{p}}(x, t)+\varepsilon \Delta T_{\mathrm{p}}(x, t)=T_{\mathrm{p}}(x, t)+\varepsilon V_{\mathrm{p}}(x, t)
\end{aligned}
$$

where $\varepsilon$ is a small real number and, as a subscript, $\varepsilon$ refers to the perturbed variables. The variation problem is obtained by introducing the following limiting process [7,22]:

$$
D_{\Delta H, \Delta W} T(x, t)=\lim _{\varepsilon \longrightarrow 0} \frac{\mathcal{O}_{\varepsilon}\left(H_{\varepsilon}, W_{\varepsilon}\right)-\mathcal{O}(H, W)}{\varepsilon}
$$

where $\mathcal{O}_{\varepsilon}\left(H_{\varepsilon}, W_{\varepsilon}\right)$ and $\mathcal{O}(H, W)$ are the operator forms of the direct problem, Equations (1)-(8), written by introducing the perturbed variables, Equations (A1)-(A4), and unperturbed variables in the direct problem. Application of the above limiting process gives the 'common' direct problem in variations expressed by

$$
\begin{aligned}
C_{\mathrm{t}} \frac{\partial V_{\mathrm{t}}(x, t)}{\partial t} & =k_{\mathrm{t}} \frac{\partial^{2} V_{\mathrm{t}}(x, t)}{\partial x^{2}}-W(t) V_{\mathrm{t}}(x, t)+\Delta W(t)\left[T_{\mathrm{a}}-T_{\mathrm{t}}(x, t)\right] \\
k_{\mathrm{t}} \frac{\partial V_{\mathrm{t}}(a, t)}{\partial x} & =k_{\mathrm{p}} \frac{\partial V_{\mathrm{p}}(a, t)}{\partial x} \\
-k_{\mathrm{t}} \frac{\partial V_{\mathrm{t}}(a, t)}{\partial x} & =H\left[V_{\mathrm{t}}(a, t)-V_{\mathrm{p}}(a, t)\right]+\Delta H\left[T_{\mathrm{t}}(a, t)-T_{\mathrm{p}}(a, t)\right]
\end{aligned}
$$




$$
\begin{aligned}
C_{\mathrm{p}} \frac{\partial V_{\mathrm{p}}(x, t)}{\partial t} & =k_{\mathrm{p}} \frac{\partial^{2} V_{\mathrm{p}}(x, t)}{\partial x^{2}} \\
-k_{\mathrm{p}} \frac{\partial V_{\mathrm{p}}(b, t)}{\partial x} & =h_{\infty} V_{\mathrm{p}}(b, t)
\end{aligned}
$$

with the initial boundary conditions

$$
\begin{aligned}
& V_{\mathrm{t}}(x, 0)=0, \quad 0 \leqslant x \leqslant a \\
& V_{\mathrm{p}}(x, 0)=0, \quad a \leqslant x \leqslant b
\end{aligned}
$$

The above variation problem will be used in the study of the stationary conditions of the augmented Lagrange functional given in Equations (A18) and (A19) in the next section.

Since the present work deals with the estimation of two unknowns, two direct problems in variations are required to compute the descent vector components, see Equations (13)-(15). They are derived, in a similar way, by considering variations in the two unknowns each at a time. In order to derive the first direct problem in variations resulting from the perturbation in $H$, we apply the following limiting process $[7,22]$ :

$$
D_{\Delta H} T(x, t)=\lim _{\varepsilon \longrightarrow 0} \frac{\mathcal{O}_{\varepsilon}\left(H_{\varepsilon}\right)-\mathcal{O}(H)}{\varepsilon}
$$

where $\mathcal{O}_{\varepsilon}\left(H_{\varepsilon}\right)$ and $\mathcal{O}(H)$ are the operator forms of the direct problem, Equations (1)-(8), written by introducing the perturbed variables, Equations (A1), (A3), and (A4), and unperturbed variables in the direct problem. Application of the above limiting process gives the first direct problem in variations expressed by Equations (16)-(23) where the subscript of the source terms $Y_{i}(x, t)$ and $Z_{i}(x, t)$ is taken as $i=1$. Second-order terms in the above limiting process are neglected.

The same procedure is used for the derivation of the second direct problem in variations resulting from the perturbation of the blood perfusion $W(t)$. Perturbed variables, i.e. Equations (A2)-(A4) are introduced in the direct problem to form the perturbed operator $\mathcal{O}_{\varepsilon}\left(W_{\varepsilon}\right)$. From the outcome result we subtract the unperturbed operator $\mathcal{O}(W)$ and applying the precedent limiting process, as shown by Equation (A13), that is

$$
D_{\Delta W} T(x, t)=\lim _{\varepsilon \longrightarrow 0} \frac{\mathcal{O}_{\varepsilon}\left(W_{\varepsilon}\right)-\mathcal{O}(W)}{\varepsilon}
$$

which leads to the second direct problem in variations given in Equations (16)-(23) where the subscript of the source terms $Y_{i}(x, t)$ and $Z_{i}(x, t)$ is taken as $i=2$.

Once the direct problems in variations are established, the next step is to calculate the variation of the functional to be minimized $J(H, W)$ denoted $\Delta J(H, W)$. This can be done with the following limit process:

$$
\begin{aligned}
\Delta J(H, W) & =\lim _{\varepsilon \longrightarrow 0} \frac{J_{\varepsilon}\left(H_{\varepsilon}, W_{\varepsilon}\right)-J(H, W)}{\varepsilon} \\
& =2 \int_{0}^{t_{\mathrm{f}}}\left[q_{\mathrm{c}}(a, t ; H, W)-q_{\mathrm{m}}(a, t)\right] \Delta q_{\mathrm{c}}(a, t ; H ; W) \mathrm{d} t
\end{aligned}
$$


The heat flux at the interface $x=a$ can be easily deduced from the direct problem in variations. First we recall that the computed heat flux at the interface can be expressed analytically by the following formula

$$
\begin{aligned}
q_{\mathrm{c}}(a, t ; H, W) & =-k_{\mathrm{t}} \frac{\partial T_{\mathrm{t}}(a, t)}{\partial x} \\
& =-k_{\mathrm{p}} \frac{\partial T_{\mathrm{p}}(a, t)}{\partial x} \\
& =H\left[T_{\mathrm{t}}(a, t)-T_{\mathrm{p}}(a, t)\right]
\end{aligned}
$$

Similarly we can show that the heat flux variation, due to the perturbation of $H$ and $W(t)$, can be expressed as

$$
\begin{aligned}
\Delta q_{\mathrm{c}}(a, t ; H, W) & =-k_{\mathrm{t}} \frac{\partial V_{\mathrm{t}}(a, t)}{\partial x} \\
& =-k_{\mathrm{p}} \frac{\partial V_{\mathrm{p}}(a, t)}{\partial x} \\
& =H\left[V_{\mathrm{t}}(a, t)-V_{\mathrm{p}}(a, t)\right]+\Delta H\left[T_{\mathrm{t}}(a, t)-T_{\mathrm{p}}(a, t)\right]
\end{aligned}
$$

These expressions represent the thermal conditions at the contact interface in the direct problem in variations established in Equations (16)-(23). The above equations contain a source term which is proportional to the temperature jump present in the direct problem statement.

\section{A.3. Adjoint problem and gradient equations}

To compute the gradient of the residual functional $J$, and the adjoint problem, the Lagrange functional is introduced. The non-null equations (1), (3), (4), (5), and (6) in the direct problem are multiplied by different Lagrange multipliers and the resulting expression is integrated over the correspondent time and space domains. Then the result is added to the functional $J(H, W)$ to yield to the following augmented functional (Lagrange functional):

$$
\begin{aligned}
\mathscr{L}= & \int_{0}^{t_{\mathrm{f}}}\left[q_{\mathrm{c}}(a, t ; H, W)-q_{\mathrm{m}}(a, t)\right]^{2} \mathrm{~d} t \\
& +\int_{0}^{a} \int_{0}^{t_{\mathrm{f}}}\left[k_{\mathrm{t}} \frac{\partial^{2} T_{\mathrm{t}}(x, t)}{\partial x^{2}}+W(t)\left[T_{\mathrm{a}}-T_{\mathrm{t}}(x, t)\right]-C_{\mathrm{t}} \frac{\partial T_{\mathrm{t}}(x, t)}{\partial t}\right] P_{\mathrm{t}}(x, t) \mathrm{d} t \mathrm{~d} x \\
& +\int_{0}^{t_{\mathrm{f}}}\left[k_{\mathrm{t}} \frac{\partial T_{\mathrm{t}}(a, t)}{\partial x}-k_{\mathrm{p}} \frac{\partial T_{\mathrm{p}}(a, t)}{\partial x}\right] Q(t) \mathrm{d} t \\
& +\int_{0}^{t_{\mathrm{f}}}\left[H\left[T_{\mathrm{t}}(a, t)-T_{\mathrm{p}}(a, t)\right]+k_{\mathrm{t}} \frac{\partial T_{\mathrm{t}}(a, t)}{\partial x}\right] U(t) \mathrm{d} t
\end{aligned}
$$




$$
\begin{aligned}
& +\int_{a}^{b} \int_{0}^{t_{\mathrm{f}}}\left[k_{\mathrm{p}} \frac{\partial^{2} T_{\mathrm{p}}(x, t)}{\partial x^{2}}-C_{\mathrm{p}} \frac{\partial T_{\mathrm{p}}(x, t)}{\partial t}\right] P_{\mathrm{p}}(x, t) \mathrm{d} t \mathrm{~d} x \\
& +\int_{0}^{t_{\mathrm{f}}}\left[h_{\infty}\left[T_{\mathrm{p}}(b, t)-T_{\infty}\right]+k_{\mathrm{p}} \frac{\partial T_{\mathrm{p}}(b, t)}{\partial x}\right] S(t) \mathrm{d} t
\end{aligned}
$$

where $P_{\mathrm{t}}(x, t), P_{\mathrm{p}}(x, t), Q(t), U(t)$, and $S(t)$ are denoted the Lagrange multipliers. Then the stationary conditions of the Lagrange functional are investigated. First let us write the variational form of the above functional, that is

$$
\begin{aligned}
\Delta \mathscr{L}= & 2 \int_{0}^{t_{\mathrm{f}}}\left[q_{\mathrm{c}}(a, t ; H, W)-q_{\mathrm{m}}(a, t)\right] \Delta q_{\mathrm{c}}(a, t ; H, W) \mathrm{d} t \\
& +\int_{0}^{a} \int_{0}^{t_{\mathrm{f}}}\left[k_{\mathrm{t}} \frac{\partial^{2} V_{\mathrm{t}}(x, t)}{\partial x^{2}}-W(t) V_{\mathrm{t}}(x, t)+\Delta W(t)\left[T_{\mathrm{a}}-T_{\mathrm{t}}(x, t)\right]-C_{\mathrm{t}} \frac{\partial V_{\mathrm{t}}(x, t)}{\partial t}\right] P_{\mathrm{t}}(x, t) \mathrm{d} t \mathrm{~d} x \\
& +\int_{0}^{t_{\mathrm{f}}}\left[k_{\mathrm{t}} \frac{\partial V_{\mathrm{t}}(a, t)}{\partial x}-k_{\mathrm{p}} \frac{\partial V_{\mathrm{p}}(a, t)}{\partial x}\right] Q(t) \mathrm{d} t \\
& +\int_{0}^{t_{\mathrm{f}}}\left[H\left[V_{\mathrm{t}}(a, t)-V_{\mathrm{p}}(a, t)\right]+\Delta H\left[T_{\mathrm{t}}(a, t)-T_{\mathrm{p}}(a, t)\right]+k_{\mathrm{t}} \frac{\partial V_{\mathrm{t}}(a, t)}{\partial x}\right] U(t) \mathrm{d} t \\
& +\int_{a}^{b} \int_{0}^{t_{\mathrm{f}}}\left[k_{\mathrm{p}} \frac{\partial^{2} V_{\mathrm{p}}(x, t)}{\partial x^{2}}-C_{\mathrm{p}} \frac{\partial V_{\mathrm{p}}(x, t)}{\partial t}\right] P_{\mathrm{p}}(x, t) \mathrm{d} t \mathrm{~d} x \\
& +\int_{0}^{t_{\mathrm{f}}}\left[h_{\infty} V_{\mathrm{p}}(b, t)+k_{\mathrm{p}} \frac{\partial V_{\mathrm{p}}(b, t)}{\partial x}\right] S(t) \mathrm{d} t
\end{aligned}
$$

In the above equation the $x$ integration operation acting on the temperature variation variables $V_{\mathrm{t}}(x, t)$ and $V_{\mathrm{p}}(x, t)$ are passed onto the corresponding adjoint variables $P_{\mathrm{t}}(x, t)$ and $P_{\mathrm{p}}(x, t)$ using integration by parts as follows:

$$
\begin{aligned}
\int_{0}^{a} \frac{\partial^{2} V_{\mathrm{t}}(x, t)}{\partial x^{2}} P_{\mathrm{t}}(x, t) \mathrm{d} x= & P_{\mathrm{t}}(a, t) \frac{\partial V_{\mathrm{t}}(a, t)}{\partial x}-P_{\mathrm{t}}(0, t) \frac{\partial V_{\mathrm{t}}(0, t)}{\partial x} \\
& -V_{\mathrm{t}}(a, t) \frac{\partial P_{\mathrm{t}}(a, t)}{\partial x}+V_{\mathrm{t}}(0, t) \frac{\partial P_{\mathrm{t}}(0, t)}{\partial x} \\
& +\int_{0}^{a} \frac{\partial^{2} P_{\mathrm{t}}(x, t)}{\partial x^{2}} V_{\mathrm{t}}(x, t) \mathrm{d} x
\end{aligned}
$$




$$
\begin{aligned}
\int_{a}^{b} \frac{\partial^{2} V_{\mathrm{p}}(x, t)}{\partial x^{2}} P_{\mathrm{p}}(x, t) \mathrm{d} x= & P_{\mathrm{p}}(b, t) \frac{\partial V_{\mathrm{p}}(b, t)}{\partial x}-P_{\mathrm{p}}(a, t) \frac{\partial V_{\mathrm{p}}(a, t)}{\partial x} \\
& -V_{\mathrm{p}}(b, t) \frac{\partial P_{\mathrm{p}}(b, t)}{\partial x}+V_{\mathrm{p}}(a, t) \frac{\partial P_{\mathrm{p}}(a, t)}{\partial x} \\
& +\int_{a}^{b} \frac{\partial^{2} P_{\mathrm{p}}(x, t)}{\partial x^{2}} V_{\mathrm{p}}(x, t) \mathrm{d} x
\end{aligned}
$$

Similarly, we perform the same integration operation with the time variable $t$ as follows:

$$
\begin{aligned}
& \int_{0}^{t_{\mathrm{f}}} \frac{\partial V_{\mathrm{t}}(x, t)}{\partial t} P_{\mathrm{t}}(x, t) \mathrm{d} x=P_{\mathrm{t}}\left(x, t_{\mathrm{f}}\right) V_{\mathrm{t}}\left(x, t_{\mathrm{f}}\right)-P_{\mathrm{t}}(x, 0) V_{\mathrm{t}}(x, 0)-\int_{0}^{t_{\mathrm{f}}} \frac{\partial P_{\mathrm{t}}(x, t)}{\partial t} V_{\mathrm{t}}(x, t) \mathrm{d} t \\
& \int_{0}^{t_{\mathrm{f}}} \frac{\partial V_{\mathrm{p}}(x, t)}{\partial t} P_{\mathrm{p}}(x, t) \mathrm{d} x=P_{\mathrm{p}}\left(x, t_{\mathrm{f}}\right) V_{\mathrm{p}}\left(x, t_{\mathrm{f}}\right)-P_{\mathrm{p}}(x, 0) V_{\mathrm{p}}(x, 0)-\int_{0}^{t_{\mathrm{f}}} \frac{\partial P_{\mathrm{p}}(x, t)}{\partial t} V_{\mathrm{p}}(x, t) \mathrm{d} t
\end{aligned}
$$

Introducing these new expressions in the variational form of the Lagrange functional and distributing the adjoint variables $P_{\mathrm{t}}(x, t)$ and $P_{\mathrm{p}}(x, z)$ and the integration operations, we obtain

$$
\begin{aligned}
\Delta \mathscr{L}= & 2 \int_{0}^{t_{\mathrm{f}}}\left[q_{\mathrm{c}}(a, t ; H, W)-q_{\mathrm{m}}(a, t)\right] \Delta q_{\mathrm{c}}(a, t ; H, W) \mathrm{d} t \\
& +k_{\mathrm{t}} \int_{0}^{t_{\mathrm{f}}}\left[P_{\mathrm{t}}(a, t) \frac{\partial V_{\mathrm{t}}(a, t)}{\partial x}-P_{\mathrm{t}}(0, t) \frac{\partial V_{\mathrm{t}}(0, t)}{\partial x}-V_{\mathrm{t}}(a, t) \frac{\partial P_{\mathrm{t}}(a, z)}{\partial x}+V_{\mathrm{t}}(0, t) \frac{\partial P_{\mathrm{t}}(0, t)}{\partial x}\right] \mathrm{d} t \\
& +C_{\mathrm{t}} \int_{0}^{a}\left[P_{\mathrm{t}}\left(x, t_{\mathrm{f}}\right) V_{\mathrm{t}}\left(x, t_{\mathrm{f}}\right)-P_{\mathrm{t}}(x, 0) V_{\mathrm{t}}(x, 0)\right] \mathrm{d} x \\
& +\int_{0}^{t_{\mathrm{f}}} \int_{0}^{a}\left[k_{\mathrm{t}} \frac{\partial^{2} P_{\mathrm{t}}(x, t)}{\partial x^{2}}+C_{\mathrm{t}} \frac{\partial P_{\mathrm{t}}(x, t)}{\partial t}-W(t) P_{\mathrm{t}}(x, t)\right] V_{\mathrm{t}}(x, t) \mathrm{d} x \mathrm{~d} t \\
& +\int_{0}^{t_{\mathrm{f}}}\left[k_{\mathrm{t}} \frac{\partial V_{\mathrm{t}}(a, t)}{\partial x}-k_{\mathrm{p}} \frac{\partial V_{\mathrm{p}}(a, t)}{\partial x}\right] Q(t) \mathrm{d} t \\
& +\int_{0}^{t_{\mathrm{f}}}\left[H\left[V_{\mathrm{t}}(a, t)-V_{\mathrm{p}}(a, t)\right]+\Delta H\left[T_{\mathrm{t}}(a, t)-T_{\mathrm{p}}(a, t)\right]+k_{\mathrm{t}} \frac{\partial V_{\mathrm{t}}(a, t)}{\partial x}\right] U(t) \mathrm{d} t \\
& +k_{\mathrm{p}} \int_{0}^{t_{\mathrm{f}}}\left[P_{\mathrm{p}}(b, t) \frac{\partial V_{\mathrm{p}}(b, t)}{\partial x}-P_{\mathrm{p}}(a, t) \frac{\partial V_{\mathrm{p}}(a, t)}{\partial x}-V_{\mathrm{p}}(b, t) \frac{\partial P_{\mathrm{p}}(b, z)}{\partial x}\right.
\end{aligned}
$$




$$
\begin{aligned}
& \left.+V_{\mathrm{p}}(a, t) \frac{\partial P_{\mathrm{p}}(a, t)}{\partial x}\right] \mathrm{d} t+C_{\mathrm{p}} \int_{a}^{b}\left[P_{\mathrm{p}}\left(x, t_{\mathrm{f}}\right) V_{\mathrm{p}}\left(x, t_{\mathrm{f}}\right)-P_{\mathrm{p}}(x, 0) V_{\mathrm{p}}(x, 0)\right] \mathrm{d} x \\
& +\int_{0}^{t_{\mathrm{f}}} \int_{0}^{a}\left[k_{\mathrm{p}} \frac{\partial^{2} P_{\mathrm{p}}(x, t)}{\partial x^{2}}+C_{\mathrm{p}} \frac{\partial P_{\mathrm{p}}(x, t)}{\partial t}\right] V_{\mathrm{p}}(x, t) \mathrm{d} x \mathrm{~d} t \\
& +\int_{0}^{t_{\mathrm{f}}}\left[h_{\infty} V_{\mathrm{p}}(b, t)+k_{\mathrm{p}} \frac{\partial V_{\mathrm{p}}(b, t)}{\partial x}\right] S(t) \mathrm{d} t
\end{aligned}
$$

By using the boundary conditions of the two direct problems in variations, Equations (16)-(23), the variational form of the Lagrange functional is reduced to the following expression:

$$
\begin{aligned}
\Delta \mathscr{L}= & \int_{0}^{a} \int_{0}^{t_{\mathrm{f}}}\left[T_{\mathrm{a}}-T_{\mathrm{t}}(x, t)\right] P_{\mathrm{t}}(x, t) \Delta W(t) \mathrm{d} t \mathrm{~d} x \\
& +\int_{0}^{t_{\mathrm{f}}}\left[T_{\mathrm{t}}(a, t)-T_{\mathrm{p}}(a, t)\right]\left[2\left[q_{\mathrm{c}}(a, t)-q_{\mathrm{m}}(a, t)\right]+P_{\mathrm{t}}(a, t)-P_{\mathrm{p}}(a, t)\right] \Delta H \mathrm{~d} t
\end{aligned}
$$

as long as the adjoint problem, expressed by Equations (31)-(38) is satisfied. When the temperatures $T_{\mathrm{t}}(x, t)$ and $T_{\mathrm{p}}(x, t)$ are solution of the direct problem, and the Lagrange multipliers $P_{\mathrm{t}}(x, t)$ and $P_{\mathrm{p}}(x, t)$ are solution of the adjoint problem, given in Equations (31)-(38), we obtain the following identity:

$$
\mathscr{L}=J(H, W) \Leftrightarrow \Delta \mathscr{L}=\Delta J(H, W)
$$

The variation of the Lagrangian functional is equivalent to the variation of the functional to be minimized. In what follows, we can determine analytically the gradient of the functional $J(H, W)$ to be minimized. By definition, and using the directional derivative defined in Equation (A13), the variation of the functional $J(H, W)$ can be written as

$$
\begin{aligned}
\Delta J(H, W)= & \lim _{\varepsilon \rightarrow 0} \frac{J(H+\varepsilon \Delta H, W+\varepsilon \Delta W)-J(H, W)}{\varepsilon} \\
= & \lim _{\varepsilon \longrightarrow 0} \frac{J(H+\varepsilon \Delta H, W)-J(H, W)}{\varepsilon} \\
& +\lim _{\varepsilon \longrightarrow 0} \frac{J(H, W+\varepsilon \Delta W)-J(H, W)}{\varepsilon} \\
= & \left\langle J_{H}^{\prime}, \Delta H\right\rangle+\left\langle J_{W}^{\prime}, \Delta W\right\rangle
\end{aligned}
$$

As we are only dealing with the linear parts of the variational form of $J(H, W)$, we can write in the right-hand side of the above equation, the gradient of the sum is equal to the sum of the gradients. Mathematically speaking, the variation $\Delta J(H, W)$ is known also as the Fréchet derivative. $J_{H}^{\prime}$ and $J_{W}^{\prime}$ are the gradients of $J(H, W)$ with respect to the heat conductance $H$ and the blood perfusion $W(t)$, respectively. Here $\langle\cdot, \cdot\rangle$ represents the defined scalar product of the working space. The working space can be $\mathbb{R}$ (real parameters), $L_{2}\left(0, t_{\mathrm{f}}\right)$ (Hilbert space) 
or $W_{2}^{i}, \quad i=1,2$ (Sobolev space) which characterize the dimensionality of the problem under investigation and the associated scalar product. Briefly, in the Hilbert space case $L_{2}$ of square integrable functions, a function $f(t)$ defined in a domain $[c, d]$ should satisfy the following properties:

$$
\int_{c}^{d}[f(t)]^{2} \mathrm{~d} t<\infty
$$

The function $f(t)$ is said to be bounded. The associated inner scalar product $\langle\cdot, \cdot\rangle$ of two functions $f(t)$ and $g(t)$ in such functional space is defined by

$$
\langle f(t), g(t)\rangle_{L_{2}}=\int_{c}^{d} f(t) g(t) \mathrm{d} t
$$

where $f(t)$ and $g(t)$ are both defined in $L_{2}(c, d)$ space. The norm of a function $f(t)$ belonging to $L_{2}\left(0, t_{\mathrm{f}}\right)$ space is a special case of the defined scalar product and given by

$$
\|f(t)\|_{L_{2}}=\left[\int_{0}^{t_{\mathrm{f}}}[f(t)]^{2} \mathrm{~d} t\right]^{1 / 2}
$$

In our case we are working in the Hilbert space $L_{2}\left(0, t_{\mathrm{f}}\right)$ and the least square norm established in the residual functional $J(H, W)$ is similar to the definition given in Equation (A30). From the above definition of the directional derivative given in Equation (A27), the scalar product, i.e. Equation (A29), and the identity equation (A26), the variation of the objective functional $J(H, W)$ can be written in the following different forms:

$$
\begin{aligned}
\Delta J(H, M)= & \left\langle J_{H}^{\prime}, \Delta H\right\rangle+\left\langle J_{W}^{\prime}, \Delta W\right\rangle \\
= & \int_{0}^{t_{\mathrm{f}}} J_{H}^{\prime} \Delta H \mathrm{~d} t+\int_{0}^{t_{\mathrm{f}}} \int_{0}^{a} J_{W}^{\prime} \Delta W(t) \mathrm{d} x \mathrm{~d} t \\
= & \int_{0}^{t_{\mathrm{f}}}\left[T_{\mathrm{t}}(a, t)-T_{\mathrm{p}}(a, t)\right]\left[2\left[q_{\mathrm{c}}(a, t)-q_{\mathrm{m}}(a, t)\right]+P_{\mathrm{t}}(a, t)-P_{\mathrm{p}}(a, t)\right] \Delta H \mathrm{~d} t \\
& +\int_{0}^{t_{\mathrm{f}}}\left[\int_{0}^{a}\left[T_{\mathrm{a}}-T_{\mathrm{t}}(x, t)\right] P_{\mathrm{t}}(x, t) \mathrm{d} x\right] \Delta W(t) \mathrm{d} t
\end{aligned}
$$

From the precedent equations and by a simple comparison of the different forms of the righthand side, one can identify the analytical expression of the gradients $J_{H}^{\prime}$ and $J_{W}^{\prime}$ in Hilbert space $L_{2}\left(0, t_{\mathrm{f}}\right)$ as given by Equations (29) and (30). The details to derive the describing equations for other possible function spaces are presented in References [6,7]. These two references provide an excellent support to derive the describing equations for a gradient-type methods to solve multi-dimensional linear and non-linear inverse heat conduction problems. As presented in the previous sections, the problem is formulated as an infinite dimensional minimization problem. The optimization problem could be transformed in finite-dimensional form by considering a parametric representation of the unknown $W(t)$ to be estimated. 


\section{ACKNOWLEDGEMENTS}

The authors would like to thank the anonymous referees for their valuable comments and help in improving the quality of this paper.

\section{REFERENCES}

1. Hadamard J. Lectures on Cauchy's Problem in Linear Differential Equations. Yale University Press: New Haven, CT, 1923.

2. Cardinali AV, Lanz O, Diller TE, Scott EP. Validation of a non-invasive thermal perfusion system using a canine medial saphenous fasciocutaneous free tissue flap model. ASME International Mechanical Engineering Congress and Exposition, Bioengineering, vol. 1. Paper No. 32354, 2002.

3. Scott EP, Robinson P, Diller TE. Development of methodologies for the estimation of blood perfusion using a minimally invasive probe (special edition). Measurement Science and Technology 1998; 9:888-897.

4. Beck JV, Blackwell B, St-Clair CR. Inverse Heat Conduction. Ill Posed Problems. Wiley: New York, 1985.

5. Oziçik MN, Orlande HRB. Inverse Heat Transfer: Fundamentals and Applications. Taylor and Francis: Pennsylvania, 1999.

6. Alifanov OM. Inverse Heat Transfer Problems. Springer: Berlin, 1994.

7. Alifanov OM, Artyukhin EE, Rumyantsev SV. Extreme Methods of Solving Ill-posed Problems with Applications to Inverse Heat Transfer Problems. Begell House: New York, 1995.

8. Morozov VA. Methods for Solving Incorrectly Posed Problems. Springer: Berlin, 1984.

9. Lions JL. Systems Governed by Partial Differential Equations. Springer: Berlin, 1971.

10. Scott EP, Robinson P, Diller TE. Estimation of blood perfusion using a minimally invasive blood perfusion probe. Advances in Heat and Mass Transfer in Biotechnology (ASME) 1997; HTD-355/BED-37: $205-212$.

11. Robinson P, Scott EP, Diller TE. Validation of methodologies for the estimation of blood perfusion using a minimally invasive probe. Advances in Heat and Mass Transfer in Biotechnology (ASME) 1998; HTD-362/BED-40:109-115.

12. Diller TE. Advances in heat flux measurements. Advances in Heat Transfer 1993; 23:279-368.

13. Pennes HH. Analysis of tissue and arterial blood temperatures in the resting human forearm. Journal of Applied Physiology 1948; 1:93-122.

14. Oziçik MN. Finite Difference Methods in Heat Transfer. CRC: Ann Arbor, 1994.

15. Loulou T, Scott EP. Thermal dose optimization in hyperthermia treatments by using the conjugate gradient method. Numerical Heat Transfer, Part A 2002; 42(7):661-683.

16. Polak E. Computational Methods in Optimization. Academic Press: New York, 1971.

17. Loulou T, Artioukhine EA. Optimal choice of descent steps in gradient type methods when applied to combined parameter and/or function estimation. Inverse Problems in Science and Engineering 2003; 11(4): 273-288.

18. Engl HW, Hanke M, Neubauer A. Regularization of Inverse Problems. Kluwer: Amsterdam, 1996.

19. Tikhonov AN, Arsenin VY. Solution of Ill-posed Problems. Winston and Sons: Washington, DC, 1977.

20. IMSL. Library Edition 10.0, User's Manual, Math/Library. IMSL, 7500 Ballaire Blvd., Houston, Texas, 1987.

21. de Boor C. A Practical Guide to Splines. Springer: Berlin, 1981.

22. Jarny Y, Ozisik MN, Bardon JP. A general optimization method using adjoint equation for solving multidimensional inverse heat conduction. International Journal of Heat and Mass Transfer 1991; 34(11): 2911-2919. 